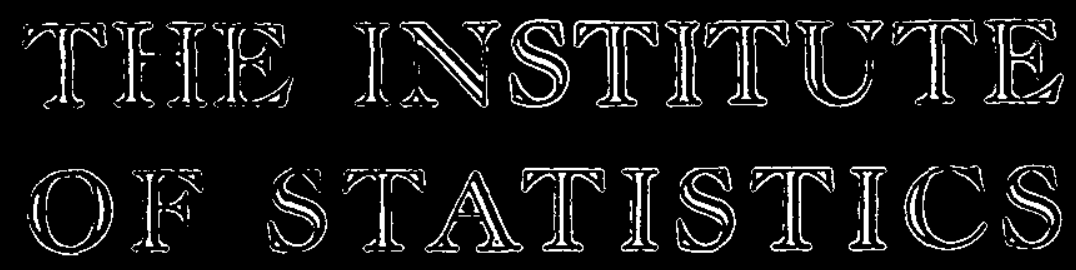

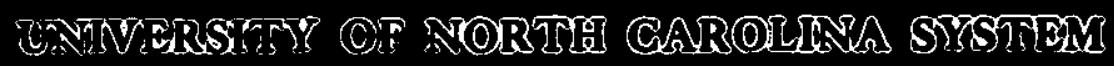

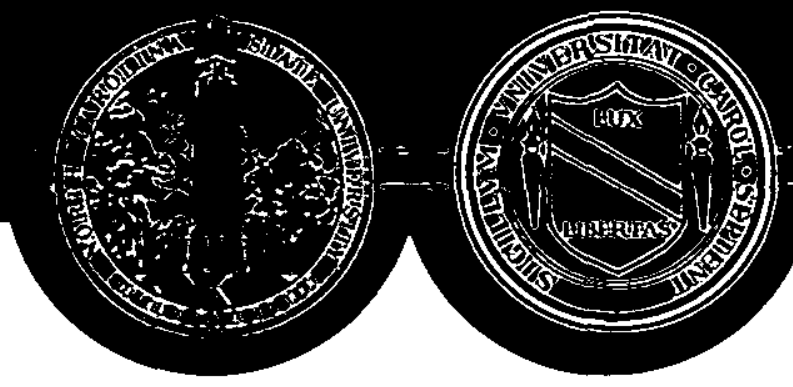

ANALYSIS OF REPEATED MEASUREMENT DATA USING THE NONLINEAR MIXED EFFECTS MODEL by

Marie Davidian and David M. Giltinan

$\div$ Institution of Statistics Mimeograph Series No. 2225 June, 1992 


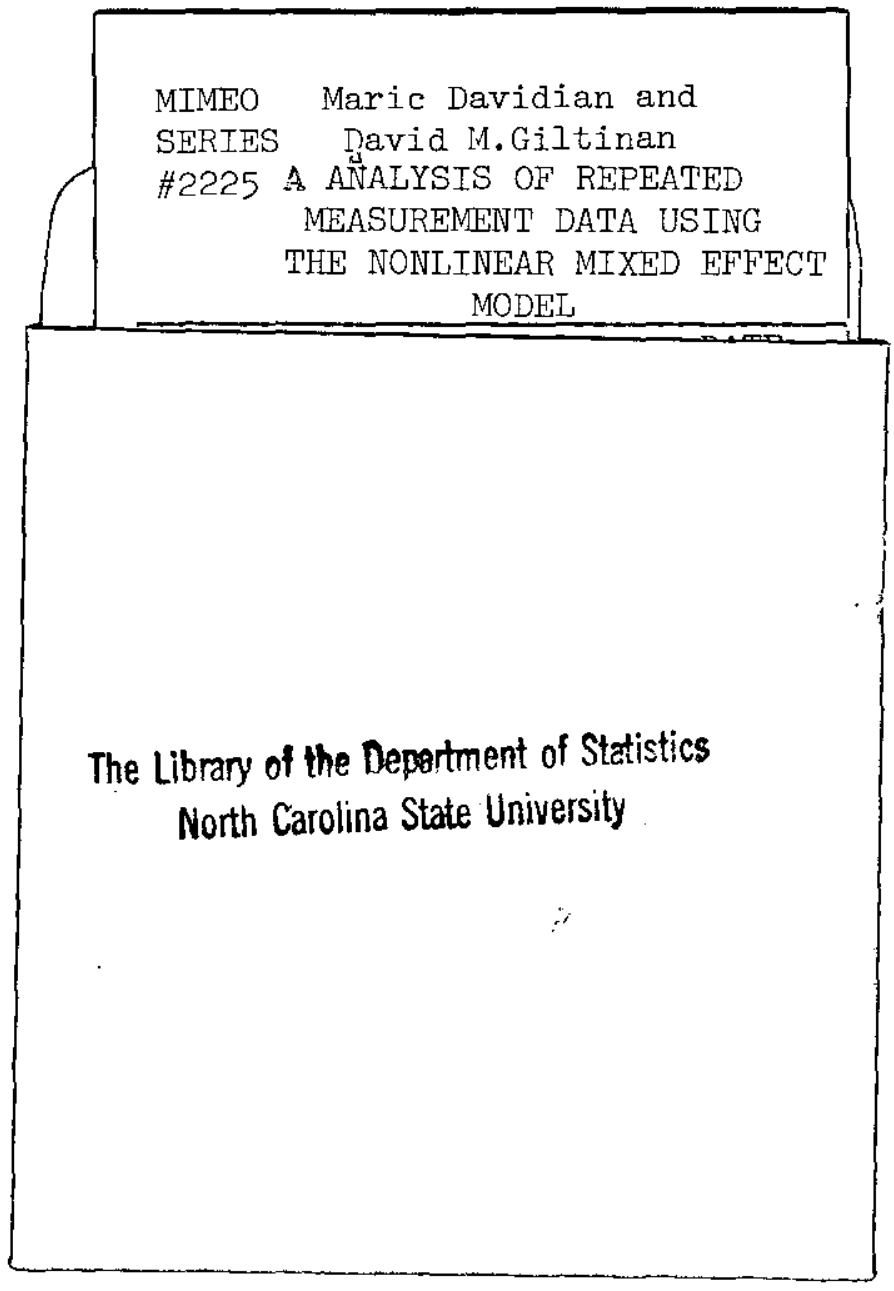

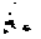




\title{
Analysis of Repeated Measurement Data Using the Nonlinear Mixed Effects Model
}

\author{
Marie Davidian* \\ Department of Statistics, Box 8209, North Carolina State University, Raleigh, NC 27695 (U.S.A.) \\ David M. Giltinan \\ Biostatistics Department, Genentech, Inc., South San Francisco, CA 94080 (U.S.A.)
}

\section{CONTENTS}

\begin{tabular}{|c|c|c|c|}
\hline \multicolumn{3}{|c|}{ Abstract } & 1 \\
\hline \multirow{3}{*}{$\begin{array}{l}1 \\
2\end{array}$} & \multicolumn{2}{|c|}{ Introduction } & 1 \\
\hline & \multicolumn{2}{|c|}{ Examples } & 2 \\
\hline & 2.1 & Bioassay for relaxin by RIA & 2 \\
\hline & 2.2 & Water transport kinetics of high flux hemodialyzers & 3 \\
\hline & 2.3 & Pharmacokinetics of cefamandole & 4 \\
\hline \multirow[t]{5}{*}{3} & \multicolumn{2}{|c|}{ The nonlinear mixed effects model } & 4 \\
\hline & 3.1 & Motivation and description & 4 \\
\hline & 3.2 & Inter-individual variability & 5 \\
\hline & 3.3 & Intra-individual variability & 8 \\
\hline & 3.4 & Summary & 8 \\
\hline \multirow[t]{5}{*}{4} & \multicolumn{2}{|c|}{ Overview of methods } & 9 \\
\hline & 4.1 & Introduction & 9 \\
\hline & 4.2 & Methods based on individual estimation & 10 \\
\hline & 4.3 & Methods based on linearization & 13 \\
\hline & 4.4 & Other methods & 17 \\
\hline \multirow[t]{4}{*}{5} & \multicolumn{2}{|c|}{ Examples revisited } & 18 \\
\hline & 5.1 & Bioassay for relaxin by RIA & 18 \\
\hline & 5.2 & Water transport kinetics of high flux hemodialyzers & 20 \\
\hline & 5.3 & Pharmacokinetics of cefamandole & 22 \\
\hline 6 & Con & usion & 24 \\
\hline \multicolumn{3}{|c|}{ References } & 24 \\
\hline
\end{tabular}

* Corresponding author 


\begin{abstract}
Situations in which repeated measurements are taken on each of several individuals arise in many areas of application. These include assay development, where dose-response data are available for each run in a series of assay experiments; pharmacokinetic analysis, where repeated blood concentration measurements are obtained from each of several subjects; and growth or decay studies, where growth or decay are measured over time for each plant, animal, or other experimental unit. In these situations, the model describing the response is often nonlinear in parameters to be estimated, as in the case of the four-parameter logistic model frequently used to characterize dose-response relationships for RIA or ELISA. Furthermore, response variability typically increases with level. Objectives of an analysis vary according to application: for assay analysis, calibration of unknowns for the most recent run may be of interest; in pharmacokinetics, characterization of drug disposition for a patient population may be the focus. The nonlinear mixed effects model has been used to describe repeated measurement data for which the mean response function is nonlinear. In this tutorial, the model is motivated and described, an overview of methods for estimation and inference in the context of the model is given, and some of the methods and analyses possible are illustrated by application to three examples from the fields of assay development, pharmacokinetics, and water transport kinetics.
\end{abstract}

\title{
1. INTRODUCTION
}

Data consisting of repeated measurements taken on each of a number of individuals arise in areas such as assay development, pharmacokinetics, and studies of growth and decay. Here, the term "individual" may refer to experiments, subjects, plants, laboratories, devices, etc., and repeated measurements on an individual may be taken over time, dose, or some other set of conditions. The relationship between response, y, and the repeated covariate, $x$, is often nonlinear in its parameters. For example, assay data frequently conform to the four-parameter logistic model

$$
\mathrm{y}=\mathrm{f}(\mathrm{x}, \beta)=\beta_{1}+\frac{\beta_{2}-\beta_{1}}{\left\{1+\exp \left[\beta_{4}\left(\log \mathrm{x}-\beta_{3}\right)\right]\right\}}
$$

where $\mathrm{x}$ is dose. Single-dose drug kinetics are commonly characterized by polyexponential functions based on compartment models, for example,

$$
\mathrm{y}=\mathrm{f}(\mathrm{x}, \beta)=\beta_{1} \exp \left(-\beta_{2} \mathrm{x}\right)+\beta_{3} \exp \left(-\beta_{4} \mathrm{x}\right)
$$

where $\mathrm{x}$ is time. A common feature in both of these contexts is a heterogeneous pattern of variability for measurements within an individual (assay run, subject) which is systematically related to overall response level.

The goals of an analysis will vary depending on the area of application. For example:

- In assay analysis, once assay procedures have stabilized, the main objective is calibration of unknown samples for the current run. Data from each run in the series contain information on the nature of intra-assay variability. Since accurate 
characterization of the pattern of intra-assay variation is crucial for appropriate calibration inference, this is an important objective in the analysis of assay data.

- In pharmacokinetic studies, repeated plasma concentration measurements are collected from subjects following single or multiple dosing. In pilot experiments on volunteers, several measurements are collected from each subject, and these are used to establish an appropriate kinetic model, obtain preliminary information on values of the pharmacokinetic parameters, and assess intra-subject measurement error, such as that due to the assay used to process blood samples. Investigation of kinetics in a more extensive patient population relies on clinical data, and only a small number of measurements are usually available from each subject. Interest in this setting focuses on estimation of "typical" values for the pharmacokinetic parameters, their relationship to individual attributes such as weight or age, and the variation in the patient population. This information may be used subsequently in the adjustment of individual dosage schedules.

In both of these settings, a goal of the analysis is to characterize inter- and intraindividual variation. Analysis should be conducted in a setting which recognizes the existence of these two sources of variation in repeated measurement data and allows them to be evaluated. A natural parametric framework which accommodates these features is the nonlinear mixed effects model. In this tutorial, we discuss this model and show how it provides a basis for inference in several application settings. In Section 2 we introduce three examples from the fields of assay development, water transport kinetics, and pharmacokinetics. Section 3 describes the model framework. An overview of approaches to estimation and inference is provided in Section 4, and some of these techniques are illustrated by application to the examples in Section 5.

\section{EXAMPLES}

\subsection{Bioassay of relaxin by $R I A$}

Table 1 shows dose-response data obtained for standard concentrations in nine runs of a bioassay for the therapeutic protein relaxin [1]. The assay is based on increased generation and release of intracellular cAMP by normal human uterine endometrial cells in the presence of relaxin. For each of the nine runs, triplicate cAMP measurements were determined by RIA for each of seven known relaxin concentrations. A single control measurement was also available for each plate. Figure 1 illustrates concentration-response data for the standard for two of the runs, where response at zero concentration has been plotted at two dilutions below the lowest standard. The four- 
parameter logistic model (1) is a reasonable representation of dose-response for a given run; however, parameters such as $\log \operatorname{ED} 50\left(\beta_{3}\right)$, background $\left(\beta_{2}\right)$, and mean response at infinite dose $\left(\beta_{1}\right)$ vary from run to run. Variability in measured cAMP levels increases with response level for a given run (see [2]) in a way that is similar across runs.

The objective of an analysis is calibration of unknowns for the most recent run. As described in [3] and [4], accuracy of calibration confidence intervals and precision profiles for a run will depend critically on how one characterizes this increasing intraassay variation. In Section 5, we will show how using the nonlinear mixed effects model as a framework for analysis allows this variation to be characterized using data from all nine runs, resulting in improved calibration inference.

\subsection{Water transport kinetics of high flux hemodialyzers}

In [5], data are presented from an experiment to evaluate the water transport kinetics of high flux membrane dialyzers used for hemodialysis for patients with end-stage renal disease. Twenty dialyzers were evaluated in vitro with bovine blood at two different blood flow rates, 200 or $300 \mathrm{ml} / \mathrm{min}$. For each of seven values of transmembrane pressure $(\mathrm{mmHg})$ exerted on the dialyzer membrane, ultrafiltration rate $(\mathrm{ml} / \mathrm{hr})$ at which water is removed was measured for each dialyzer. These data are given in Table 2, and profiles for each dialyzer are plotted in Figure 2.

Since the relationship is governed by protein polarization, which causes a relatively constant ultrafiltration rate at high pressure, and oncotic pressure, a nonlinear model for the relationship between ultrafiltration rate and transmembrane pressure $x$ is appropriate [5]:

$$
f(x, \beta)=\beta_{1}\left\{1-\exp \left[-\beta_{2}\left(x-\beta_{3}\right)\right]\right\}
$$

where $\beta_{1}$ is the maximum attainable ultrafiltration rate due to protein polarization, $\beta_{2}$ is a hydraulic permeability transport rate, and $\beta_{3}$ is the transmembrane pressure required to offset patient oncotic pressure. For a given dialyzer, the seven available observations provide enough information to fit (3) to the data for that dialyzer by nonlinear (unweighted) least squares (LS). Figure 3 shows a plot of LS residuals against predicted response, as described in [3], for a typical dialyzer. The "fan-shaped" pattern is evidence that intra-dialyzer variability is an increasing function of ultrafiltration response level.

One objective of the experiment was to contrast kinetic properties for dialyzers at the two different blood flow rates. In Section 5 we report the results of an analysis to investigate this question based on the nonlinear mixed effects model which allows 
incorporation of inter- and intra-dialyzer variation.

\subsection{Pharmacokinetics of cefamandole}

The results of a pilot study to investigate the pharmacokinetics of cefamandole, a cephalosporin antibiotic, are reported in [6] and are shown here in Table 3. A dose of 15 $\mathrm{mg} / \mathrm{kg}$ body weight of cefamandole was administered by 10 minute intravenous infusion to six healthy male volunteers, and blood samples were collected from each subject at each of fourteen time points post-dose. Plasma levels $(\mu \mathrm{g} / \mathrm{ml})$ for each sample were determined by HPLC. Figure 4 plots the resulting plasma level-time profiles for each subject and indicates that the biexponential function (2) arising from a twocompartment model for cefamandole kinetics is reasonable, although the values of the pharmacokinetic parameters may vary across subjects. For pharmacokinetic data, the variation associated with plasma concentrations is likely to be an increasing function of level, in part due to the nature of the HPLC assay $[7,8,9]$.

As discussed in Section 1, the goals of an analysis of data from a pilot study such as this are to characterize this intra-subject variation, which is likely to be the similar for all individuals, and to estimate the pharmacokinetic parameters. We illustrate these ideas in Section 5.

\section{THE NONLINEAR MIXED EFFECTS MODEL}

\subsection{Motivation and description}

In the three examples in Section 2, several common features are apparent: The same nonlinear model for the relationship between response and $\mathrm{x}$ is suitable for describing data for each individual, but the values of the parameters that specify the model fully may differ across individuals (inter-individual variation). Furthermore, the variability associated with response measurements for a given individual depends on response level in a way that is likely to be similar for all individuals, due to, for example, properties of an assay (intra-individual variation). Correct analysis should account for both of these features; moreover, characterization of these features may be a goal. The following nonlinear mixed effects model incorporates both sources of variation. The use of this model as an analytic tool was pioneered by Beal and Sheiner $[7,8,9]$, who recognized and advocated the need to accommodate and assess both types of variability in pharmacokinetic analysis. More general specifications than that presented here are possible, and the reader is referred to the work of Beal and Sheiner for such formulations with particular reference to pharmacokinetics.

Let $\mathrm{y}_{i j}$ denote the $j$ th response, $j=1, \ldots, \mathrm{m}_{i}$, for the $i$ th individual, $i=1, \ldots, \mathbf{n}$, at 
the set of conditions summarized by the vector of covariates $x_{i j}$. The vector $x_{i j}$ incorporates variables such as time, dose, pressure, etc. Suppose that a (nonlinear) function $\mathrm{f}(\mathrm{x}, \beta)$ may be specified to model the relationship between $\mathrm{y}_{i j}$ and $\mathrm{x}_{i j}$.

Inter-individual variability is accommodated by the assumption that, although $f$ is common to all individuals, the $(\mathrm{p} \times 1)$ regression parameter vector $\beta$ may vary across individuals. This is incorporated by specification of a separate $(p \times 1)$ vector of parameters $\beta_{i}$ for the $i$ th individual. For example, for the relaxin bioassay, $\beta_{i}$ would be the $(4 \times 1)$ vector whose components are the parameters of the four-parameter logistic model (1) corresponding to the $i$ th run of the assay. The mean response for individual $i$, given the parameter vector $\beta_{i}$, is thus $\mathrm{E}\left(\mathrm{y}_{i j} \mid \beta_{i}\right)=\mathrm{f}\left(\mathrm{x}_{i j}, \beta_{i}\right)$.

For a given individual, the variability in $y_{i j}$ may be a function of $f\left(x_{i j}, \beta_{i}\right)$. For example, variance may be proportional to a power of the mean response given $\beta_{i}$, that is, $\operatorname{Var}\left(\mathrm{y}_{i j} \mid \beta_{i}\right)=\sigma^{2}\left\{\mathrm{f}\left(\mathrm{x}_{i j}, \beta_{i}\right)\right\}^{2 \theta}$ for some scale parameter $\sigma$ and a power $\theta$; if $\theta=1$, this is the constant coefficient of variation (CV) model with $\mathrm{CV}=\sigma$. In this specification, the parameters $\sigma$ and $\theta$ are common to all individuals, reflecting the belief that the pattern of variability in measurements is similar across individuals. This will be the case if, for example, the pattern is primarily due to a (common) assay used to obtain response measurements. In general, write $\operatorname{Var}\left(\mathrm{y}_{i j} \mid \beta_{i}\right)=\sigma^{2} \mathrm{~g}^{2}\left\{\mathrm{f}\left(\mathrm{x}_{i j}, \beta_{i}\right), \theta\right\}$, where the variance function $\mathrm{g}$ describes the common pattern of variability. Other examples of models $\mathrm{g}$ for intra-individual variance are given in [3] and [9].

With these definitions, the nonlinear mixed effects model assumes that the jth measurement on individual $i$ can be written as

$$
\mathrm{y}_{i j}=\mathrm{f}\left(\mathrm{x}_{i j}, \beta_{i}\right)+\sigma \mathrm{g}\left\{\left\{\mathrm{f}\left(\mathrm{x}_{i j}, \beta_{i}\right), \theta\right\} \epsilon_{i j}\right.
$$

where $\epsilon_{i j}$ is a random error with mean 0 and variance 1 . We now turn our attention to further description of the components of the model.

\subsection{Inter-individual variability}

In (4), inter-individual variation is modeled through the assumption of the individualspecific regression parameter vector $\beta_{i}$. Part of the inter-individual variation in the values of the parameters characterizing mean response may be due to systematic dependence on individual attributes. For example, pharmacokinetic parameters are well-known to depend on an individual's weight, disease status, and so on $[7,8]$. Parameters may also vary due to unexplained random variation in the population of individuals; for example, due to natural biological variation among subjects or subtle 
run-to-run variation in assay procedure.

To account for these possibilities, a model for the dependence of $\beta_{\boldsymbol{i}}$ on individual attributes and random variation may be specified. The simplest such model is that in which inter-individual variation is assumed to be entirely due to unexplained phenomena:

$$
\beta_{i}=\gamma+z_{i},
$$

where $\gamma$ is a $(p \times 1)$ vector of fixed parameters and $z_{i}$ is a random vector assumed to arise from a population with mean $0_{p}, a(p \times 1)$ vector of zeros, and covariance matrix $\Sigma$. Equation (5) states that the $\beta_{i}$ vary in the population of individuals about the value $\gamma$, their mean, and the variation in the population is described by the matrix $\Sigma$. The diagonal elements of $\Sigma$ characterize the variance of each component of the $\beta_{i}$ about $\gamma$, and the off-diagonal elements describe how the components vary together (covariance).

A more complicated model allows for dependence on both random and systematic phenomena. For example, in the dialyzer study of Section 2.2, water transport kinetics may be thought to vary among dialyzers in part because of flow rate and in part because of natural variation expected to occur among the devices. Both possibilities can be taken into account by assuming that

$$
\begin{aligned}
& \beta_{i}=\gamma_{200}+z_{i} \text { if dialyzer } i \text { is used with flow rate } 200 \mathrm{ml} / \mathrm{min}, \\
& \beta_{i}=\gamma_{300}+z_{i} \text { if dialyzer } i \text { is used with flow rate } 300 \mathrm{ml} / \mathrm{min},
\end{aligned}
$$

where $\gamma_{200}$ and $\gamma_{300}$ are $(3 \times 1)$ vectors describing the central tendency of the 3 kinetic parameters in (3) for the populations of dialyzers operated at flow rates $200 \mathrm{ml} / \mathrm{min}$ and $300 \mathrm{ml} / \mathrm{min}$, respectively. The random component $z_{i}$ is again assumed to have zero mean and covariance $\Sigma$, so that random variation in both populations is assumed to be similar. Model (6) may be written compactly in the form of a "linear regression" model:

$$
\beta_{i}=\mathrm{W}_{i} \gamma+\mathrm{z}_{i}
$$

where $\gamma=\left[\gamma_{200}^{\mathrm{T}}, \gamma_{300}^{\mathrm{T}}\right]^{\mathrm{T}}$, that is, the $(6 \times 1)$ vector with $\gamma_{200}$ and $\gamma_{300}$ stacked, and $\mathrm{W}_{i}$ is a $(3 \times 6)$ "design" matrix such that

$$
\begin{array}{ll}
\mathrm{W}_{i}=\left[\mathrm{I}_{3} \mid 0_{3,3}\right] & \text { if dialyzer } i \text { is used with flow rate } 200 \mathrm{ml} / \mathrm{min} \\
\mathrm{W}_{i}=\left[0_{3,3} \mid \mathrm{I}_{3}\right] & \text { if dialyzer } i \text { is used with flow rate } 300 \mathrm{ml} / \mathrm{min}
\end{array}
$$


where $\mathrm{I}_{r}$ is a $(r \times r)$ identity matrix and $0_{r, s}$ is a $(r \times s)$ matrix of zeros. Note that, under model (7), a comparison of kinetics between the 2 flow rates could be made by comparing $\gamma_{200}$ and $\gamma_{300}$, the "typical" kinetic parameters for each population.

As another example, consider a situation in which pharmacokinetic parameters are thought to depend systematically on individual attributes. For specificity, suppose that $\beta_{i}$ is a $(2 \times 1)$ vector whose components are clearance $C L_{i}$ and apparent volume of distribution $V_{i}$. Suppose that clearance is thought to be a linear function of an individual's weight $W_{i}$. Random variation in pharmacokinetic parameters often increases with their magnitude $[7,8]$. These features are accommodated by the following models for $C L_{i}$ and $V_{i}$ :

$$
C L_{i}=\left(\gamma_{1}+\gamma_{2} \mathrm{~W}_{i}\right) \exp \left(\mathrm{z}_{i 1}\right) ; \quad V_{i}=\gamma_{3} \exp \left(\mathrm{z}_{\mathrm{i} 2}\right),
$$

where $\gamma=\left[\gamma_{1}, \gamma_{2}, \gamma_{3}\right]^{\mathrm{T}}$ and $z_{i}=\left[z_{i 1}, z_{i 2}\right]^{T}$. If the components of the random vector $z_{i}$ have symmetrically-shaped distributions and $z_{i}$ is centered at 0 with covariance $\Sigma$, then the distributions of $C L_{i}$ and $V_{i}$ will be skewed with constant coefficient of variation. To illustrate further, suppose that $\beta_{i}$ contains a third component $k a_{i}$, the first-order absorption rate constant, but there is reason to believe that this parameter varies little across individuals. This feature may lead to the assumption that $k a_{i}$ is fixed across individuals, so a specification for $k a_{i}$ should include no random variation:

$$
k a_{i}=\gamma_{4}
$$

Writing $\gamma=\left[\gamma_{1}, \gamma_{2}, \gamma_{3}, \gamma_{4}\right]^{\mathrm{T}}$ and $z_{i}=\left[z_{i 1}, z_{i 2}\right]^{\mathrm{T}}$, the model for $\beta_{i}=\left[C L_{i}, V_{i}, k a_{i}\right]^{\mathrm{T}}$ may be written compactly as

$$
\beta_{i}=\mathrm{h}\left(\mathrm{W}_{i}, \gamma, \mathrm{z}_{i}\right)
$$

where $\mathrm{h}$ is the trivariate-valued function given in (9) and (10). Note that because $k a_{i}$ is assumed to be fixed, the dimensions of $\gamma$ and $z_{i}$ need not be the same.

Equation (11) is a general model describing the variation in parameter values across individuals. We shall use this notation for general models for $\beta_{i}$, where $\mathrm{W}_{i}$ is a vector or matrix containing information on individual attributes such as weight or group, and, in accord with usage in the pharmacokinetics literature, refer to $h$ as the inter-individual regression model. Note that this general specification subsumes simpler ones such as (5) and (7). Under such a specification, the $\beta_{i}$ themselves are random vectors. 


\subsection{Intra-individual variability}

In the nonlinear mixed effects model (4), intra-individual variation is modeled through the intra-individual variance function $\mathrm{g}$, the parameters $\sigma$ and $\theta$, and the random errors $\epsilon_{i j}$. This variation is interpreted as that associated with measurements on a given individual $i$. It is often reasonable to assume that measurements within a given individual are statistically independent, reflected in the model by the assumption that the random errors $\epsilon_{i j}$ are independently distributed.

In some settings, this assumption may not be realistic. When measurements are taken on a given individual at closely space time points, it is likely that successive measurements may be correlated; that is, responses taken close in time may be "more alike" than those taken far apart. This situation may be accommodated by assuming that the $\epsilon_{i j}$ are not statistically independent but are correlated in some specifiable way. An example is to assume that the random errors follow an autoregressive process of order 1 ; see [10, ch. 6]. In practice, however, it is often assumed that the $\epsilon_{i j}$ are independent, in part because successive time points are thought to be sufficiently far apart so that such serial correlation is negligible. Another justification is that, in (4), the fact that the common (random) $\beta_{i}$ appears in $f$ and $g$ will have the effect of inducing a relationship among measurements on individual $i$. This is considered to be adequate to account for any association that may actually exist among measurements on an individual [11].

Based on these considerations, we assume for our discussion that the $\epsilon_{i j}$ are independent. The methods for estimation in the nonlinear mixed effects model in Section 4 may be generalized to the case of correlated $\epsilon_{i j}$; see [1] for details.

\subsection{Summary}

For convenience, we summarize in vector notation the version of the nonlinear mixed effects model that forms the basis for our discussion. Let $\mathrm{y}_{i}=\left[\mathrm{y}_{i 1}, \ldots, \mathrm{y}_{i m_{i}}\right]^{\mathrm{T}}, \mathrm{f}_{i}\left(\beta_{i}\right)=$ $\left[\mathrm{f}\left(\mathrm{x}_{\mathrm{i1}}, \beta_{i}\right), \ldots, \mathrm{f}\left(\mathrm{x}_{i m_{i}}, \beta_{i}\right)\right]^{\mathrm{T}}$, and $\epsilon_{i}=\left[\epsilon_{i 1}, \ldots, \epsilon_{i m_{i}}\right]^{\mathrm{T}}$ be the $\left(\mathrm{m}_{i} \times 1\right)$ vectors of responses, mean response functions, and random errors, respectively, for individual i. Let $\mathrm{G}_{\boldsymbol{i}}\left(\beta_{\boldsymbol{i}}, \theta\right)$ be the $\left(\mathrm{m}_{i} \times \mathrm{m}_{i}\right)$ diagonal matrix with diagonal elements $\mathrm{g}_{i j}=\mathrm{g}\left\{\mathrm{f}\left(\mathrm{x}_{i j}, \beta_{i}\right), \theta\right\}$. With these definitions, we write the nonlinear mixed effects model as

$$
\begin{gathered}
\mathrm{y}_{i}=\mathrm{f}_{i}\left(\beta_{i}\right)+\sigma \mathrm{G}_{i}\left(\beta_{i}, \theta\right) \epsilon_{i}\left(\mathrm{~m}_{i} \times 1\right), \\
\beta_{i}=\mathrm{h}\left(\mathrm{W}_{i}, \gamma, \mathrm{z}_{i}\right)(\mathrm{p} \times 1), \\
\mathrm{z}_{\mathrm{i}} \sim(0, \Sigma)(M \times 1) \text { distributed independently of } \epsilon_{i} \sim\left(0, \mathrm{I}_{m_{i}}\right)\left(\mathrm{m}_{i} \times 1\right), \\
i=1, \ldots, \mathrm{n}, \quad j=1, \ldots, \mathrm{m}_{i},
\end{gathered}
$$


where the notation " $(a, B)$ " means "distributed with mean $a$ and covariance matrix $B, " M$ is the dimension of the random components $z_{i}$ for the inter-individual regression model $\mathrm{h}$, and the assumption that $z_{i}$ and $\epsilon_{i}$ are distributed independently reflects the belief that the mechanisms governing the 2 sources of variation operate independently. This is a version of the model discussed by several authors $[4,5,7,8,11,12]$.

\section{OVERVIEW OF METHODS}

\subsection{Introduction}

In the context of the nonlinear mixed effects model (12), questions of interest may be formulated in terms of the components of the model. In assay analysis, for example, the objective is individual inference for a given run $i$; in particular, this involves estimation of $\beta_{i}$ characterizing the standard curve for run $i$ and estimation of $\theta$ (which will determine the weighting scheme for this fit), calibration of unknown samples based on this estimate of $\beta_{i}$, and construction of calibration confidence intervals and precision profiles (which will depend on the estimates for $\beta_{i}, \sigma$, and $\theta$ ). In pharmacokinetic analysis, one goal is population inference; this involves determination of an appropriate function $\mathrm{h}$ and estimation of $\gamma$ and $\Sigma$, all of which characterize the population of subjects. Another aim in pharmacokinetic analysis is individual inference-estimation of $\beta_{i}$ for a particular individual. Under the model, it seems reasonable to expect that the information available from all individuals will be useful for both population and individual inference. This is in fact the case-estimates of $\beta_{i}$ and $\theta$, for example, that make use of information from all subjects are preferred to those based on data from individual $i$ alone [4].

The model (12) acknowledges that inter- and intra-individual variation should be considered in an analysis of repeated measurement data. As pointed out by Beal and Sheiner [7], early attempts at analysis of these data did not take both features into account. In particular, the popular method of analysis was essentially to assume that the model for $\mathrm{y}_{i j}$ is

$$
\mathrm{y}_{i j}=\mathrm{f}\left(\mathrm{x}_{i j}, \beta\right)+\sigma \epsilon_{i j}
$$

where $\beta$ is common to all individuals. Estimation of $\beta$, the vector of model parameters (assumed the same for all individuals), was accomplished by ordinary nonlinear LS based on (13), "pooling" the data from all individuals. This is referred to as the "naive pooled data" method [7,8], since it ignores variation across individuals. Estimates of $\beta$ obtained by this method can be very biased or imprecise [8], and no assessment of inter- 
individual variability is possible. This method is not recommended [8].

The nonlinear mixed effects model (12) is complex, since, in order to account for the two sources of variation, two random components, $z_{i}$ and $\epsilon_{i}$, are required. The random component $z_{i}$ appears in the model through the nonlinear functions $h$ and $f$; thus, the effect of inter-individual variation on response measurements is complicated. Standard statistical methodology such as maximum likelihood (ML) estimation or LS is predicated on the ability to specify a distributional model for a response vector $y_{i}$. In this situation, because of the complex way in which $z_{i}$ appears in the model, it is not possible to write down a distribution for $\mathrm{y}_{i}$, even if it is assumed that both $z_{i}$ and $\epsilon_{i}$ are normally distributed. Thus, standard techniques may be difficult to implement. As a result, many of the methods that have been proposed for analysis of (12) are based on approximations which allow a distribution to be specified for $\mathrm{y}_{i}$.

We now review some methods for estimation of $\gamma, \Sigma, \beta_{i}, \sigma$, and $\theta$ for the nonlinear mixed effects model (12) that have been suggested in the literature. The methods we describe represent only some of procedures that have been proposed, and references to other methods are given.

\subsection{Methods based on individual estimates}

One approach to an approximation based on (12) is based on the ability to construct, for each individual, estimates $\beta_{i}^{*}$ for $\beta_{i}$. These estimates form the basis for estimation of $\gamma, \Sigma, \sigma, \theta$ and, in some cases, improved estimates for $\beta_{i}$. Intuitively, for this idea to be successful, sufficient data must be available on each individual for suitable $\beta_{i}^{*}$ to be obtained. These methods are referred to as two-stage methods in the pharmacokinetics literature $[7,8,12]$ since the idea consists of two stages: construction of $\beta_{i}^{*}$ and subsequent estimation of other parameters.

The standard two-stage (STS) method [for example, 12] treats estimates $\beta_{i}^{*}$ as if they were the true $\beta_{i}$. In the simplest case of (5), estimates for $\gamma$ and $\Sigma$ would be constructed as

$$
\hat{\gamma}=\mathrm{n}^{-1} \sum_{i=1}^{\mathrm{n}} \beta_{i}^{*} ; \quad \hat{\Sigma}=(\mathrm{n}-1)^{-1} \sum_{i=1}^{n}\left(\beta_{i}^{*}-\hat{\gamma}\right)\left(\beta_{i}^{*}-\hat{\gamma}\right)^{\mathrm{T}}
$$

the sample mean and covariance of $\gamma$ and $\Sigma$, respectively. Although simple, these estimators take no account of the uncertainty associated with estimation of $\beta_{i}$ by $\beta_{i}^{*}$. The result is that the estimator for $\Sigma$ can be very biased and imprecise. Furthermore, no attempt to improve on $\beta_{i}^{*}$ by taking advantage of all available information is made. These estimators are generally regarded as undesirable $[4,7,8,12]$.

The problems with STS estimation point out the need to take estimation error for $\beta_{i}^{*}$ 
into account. Several methods to do this have been proposed $[7,8,12,13]$; we describe explicitly one such method, referred to in [12] as the global two-stage (GTS) method. The method is given in terms of a linear inter-individual regression model as in (7).

An assessment of the uncertainty of estimation in $\beta_{i}^{*}$ may be obtained by appealing to large sample theory for nonlinear regression, as described in [3, sec. 3.2]. The theory states that, for large numbers of observations (in our case, large $\mathrm{m}_{i}$ ), the sampling distribution of an estimator $\beta_{i}^{*}$ is $\mathrm{p}$-variate normal with mean $\beta_{i}$ and some covariance matrix $V_{i}$, say, where the form of $V_{i}$ is determined by the nature of $f$ and $\beta_{i}^{*}$ and depends on $\beta_{i}$. In practice, $V_{i}$ is estimated by replacing parameters by estimates where they appear; henceforth, assume that $V_{i}$ refers to such an estimate. If this theory is relevant, we have that, given $\beta_{i}, \beta_{i}^{*}$ is approximately normally distributed with mean $\beta_{i}$ and covariance $\mathrm{V}_{i}$, write $\beta_{i}^{*} \mid \beta_{i} \sim \mathrm{N}\left(\beta_{i}, \mathrm{~V}_{i}\right)$. If we are further willing to assume that $\beta_{i}$ is normally distributed with mean $\mathrm{W}_{i} \gamma$ and covariance $\Sigma$, as suggested by (7) for normal $z_{i}$, then it follows that $\beta_{i}^{*} \sim \mathrm{N}\left(\mathrm{W}_{i} \gamma, \mathrm{V}_{i}+\Sigma\right)$. This argument thus leads to a distributional assumption about $\beta_{i}^{*}$, suggesting that standard approaches to estimation of $\gamma$ and $\Sigma$ may be used, treating the $\beta_{i}^{*}$ as "data." The GTS method estimates $\gamma$ and $\Sigma$ by ML estimation based on this normal distribution and is implemented by a two-step iterative algorithm $[1,12]$ which produces as a byproduct "refined" estimates of $\beta_{i}$ that make use of information from all individuals. The algorithm may be started by using initial values for $\gamma$ and $\Sigma$ from (14). At iteration $(k+1)$ :

(1) Produce refined estimates of $\beta_{i}$ :

$$
\hat{\beta}_{i}^{(k+1)}=\left(\mathrm{V}_{i}^{-1}+\hat{\Sigma}_{(k)}^{-1}\right)^{-1}\left(\mathrm{~V}_{i}^{-1} \beta_{i}^{*}+\hat{\Sigma}_{(k)}^{-1} \mathrm{~W}_{i} \hat{\gamma}_{(k)}\right) \text { for } i=1, \ldots, \mathrm{n}
$$

(2) Obtain updated estimates of $\gamma$ and $\Sigma$ :

$$
\begin{gathered}
\hat{\gamma}_{(k+1)}=\sum_{i=1}^{n} \mathrm{Q}_{i}^{(k)} \hat{\beta}_{i}^{(k+1)}, \quad \mathrm{Q}_{i}^{(k)}=\left(\sum_{i=1}^{n} \mathrm{~W}_{i}^{\mathrm{T}} \hat{\Sigma}_{(k)}^{-1} \mathrm{~W}_{i}\right)^{-1} \mathrm{~W}_{i}^{\mathrm{T}} \hat{\Sigma}_{(k)}^{-1} \\
\hat{\Sigma}_{(k+1)}=\mathrm{n}^{-1} \sum_{i=1}^{n}\left(\hat{\beta}_{i}^{(k+1)}-\mathrm{W}_{i} \hat{\gamma}_{(k+1)}\right)\left(\hat{\beta}_{i}^{(k+1)}-\mathrm{W}_{i} \hat{\gamma}_{(k+1)}\right)^{\mathrm{T}} \\
+\mathrm{n}^{-1} \sum_{i=1}^{n}\left(\mathrm{~V}_{i}^{-1}+\hat{\Sigma}_{(k)}^{-1}\right)^{-1}
\end{gathered}
$$

Iteration continues until the algorithm converges to final estimates $\hat{\gamma}, \hat{\Sigma}$, and $\hat{\beta}_{i}$. Note that the "refined" estimates in (15) have the form of a "weighted average" of the individual estimate $\beta_{i}^{*}$ obtained from the data for individual $i$ only and $\mathrm{W}_{i} \hat{\gamma}$, the estimate of $\beta_{i}$ predicated on (7) using data from all individuals. These estimates are in 
fact empirical Bayes estimates for $\beta_{i}$; that is, $\hat{\beta}_{i}$ is the mean of the distribution of $\beta_{i}$ given the data $\left\{\mathrm{y}_{i j}\right\}$, where $\gamma$ and $\Sigma$ have been replaced by the current estimates [12].

By standard statistical theory for ML estimation, estimates of the uncertainty associated with, for example, $\hat{\gamma}$, may be obtained. An estimate of the covariance matrix for $\hat{\gamma}$ is

$$
\hat{\Delta}=\left\{\sum_{i=1}^{n} \mathrm{~W}_{i}^{\mathrm{T}}\left(\mathrm{V}_{i}^{-1}+\hat{\Sigma}^{-1}\right)^{-1} \mathrm{~W}_{i}\right\}^{-1}
$$

([1]) and may be used to construct hypothesis tests about $\gamma$, as described in Section 5 .

Two-stage methods are based on separate estimates $\beta_{i}^{*}$ for each individual. Thus, it is not straightforward to use these methods when one of the components of $\beta_{i}$ is taken to remain constant across individuals, as in the pharmacokinetic example (10).

For any two-stage method, the quality of the estimates of $\gamma$ and $\Sigma$ will depend on the quality of the estimates $\beta_{i}^{*}$. If intra-individual variance is not constant but varies according to the function $g$ with response level, using ordinary LS estimates for $\beta_{i}^{*}$ will be undesirable, because LS estimates will be inefficient relative to estimates based on weighted least squares (WLS) [3]. The weighting scheme to be used will depend on what is known about the function $\mathrm{g}$. As is frequently the case, one may be able to specify a function $g$ which describes the pattern of variation, but the value of $\theta$ providing a full characterization is unknown and must be estimated from the data [3]. If it is realistic to assume a common pattern of variation for all individuals, as in (12), it makes sense to use the data from all individuals to estimate $\theta$ (and $\sigma$ ) rather than to estimate separate values for each individual.

This idea is the basis for the following proposal for obtaining $\beta_{i}^{*}$ advocated in [4]. We first review estimation for data from a single individual. As described in [3], for a given individual, variance function estimation procedures use residuals from a previous fit as the basis for estimation of $\sigma$ and $\theta$. Two such procedures are described in [3, sec. 4] and are incorporated into an iterative generalized least squares (GLS) algorithm for estimation of the individual's regression parameter. This algorithm may be summarized as follows for use with data from a single individual. For individual $i$ :

1. Obtain the LS estimator $\beta_{i}^{*(0)}$ and set $k=0$.

2. Given $\beta_{i}^{*(k)}$, minimize the objective function $O_{i}\left(\beta_{i}^{*(k)}, \sigma, \theta\right)$ in $\sigma$ and $\theta$ to obtain $\hat{\theta}^{(k)}$, where $\mathrm{O}_{i}\left(\beta_{i}, \sigma, \theta\right)$ is an objective function such as

$$
\mathrm{PL}_{i}\left(\beta_{i}, \sigma, \theta\right)=\sum_{j=1}^{m_{i}}\left(\frac{\left\{\mathrm{y}_{i j}-\mathrm{f}\left(\mathrm{x}_{i j}, \beta_{i}\right)\right\}^{2}}{\sigma^{2} \mathrm{~g}^{2}\left\{\mathrm{f}\left(\mathrm{x}_{i j}, \beta_{i}\right), \theta\right\}}+\log \left[\sigma^{2} \mathrm{~g}^{2}\left\{\mathrm{f}\left(\mathrm{x}_{i j}, \beta_{i}\right), \theta\right\}\right]\right),
$$




$$
\mathrm{AR}_{i}\left(\beta_{i}, \sigma, \theta\right)=\sum_{j=1}^{m_{i}}\left(\frac{\left|\mathrm{y}_{i j}-\mathrm{f}\left(\mathrm{x}_{i j}, \beta_{i}\right)\right|}{\sigma \mathrm{g}\left\{\mathrm{f}\left(\mathrm{x}_{i j}, \beta_{i}\right), \theta\right\}}+\log \left[\sigma \mathrm{g}\left\{\mathrm{f}\left(\mathrm{x}_{i j}, \beta_{i}\right), \theta\right\}\right]\right)
$$

and form estimated weights $\hat{\mathrm{w}}_{i j}^{(k)}=1 / \mathrm{g}^{2}\left\{\mathrm{f}\left(\mathrm{x}_{i j}, \beta_{i}^{*(k)}\right), \hat{\theta}^{(k)}\right\}$.

3. Obtain the GLS estimator of $\beta_{i}$ by WLS with weights $\hat{w}_{i j}^{(k)}$. Set $k=k+1$, let $\beta_{i}^{*(k)}$ be this GLS estimator, and return to step 2.

The objective functions for estimation of $(\sigma, \theta)$ in step 2 are motivated in [3,4]. The scheme may be iterated a fixed number of times or until convergence, see [3].

In [4], the GLS algorithm is extended to allow estimation of $\sigma$ and $\theta$ based on data from all individuals:

1. For each individual $i$, estimate $\beta_{i}$ by LS. Call these $\mathrm{n}$ estimates $\beta_{i}^{*(0)}, i=1, \ldots, \mathrm{n}$.

2. Minimize in $\sigma$ and $\theta$

$$
\sum_{i=1}^{n} O_{i}\left(\beta_{i}^{*(k)}, \sigma, \theta\right)
$$

where $O_{i}$ is one of the objective functions in (17), obtaining $\hat{\theta}^{(k)}$. For each individual $i$, form estimated weights $\hat{\mathrm{w}}_{i j}^{(k)}=1 / \mathrm{g}^{2}\left\{\mathrm{f}\left(\mathrm{x}_{i j}, \beta_{i}^{*(k)}\right), \hat{\theta}^{(k)}\right\}, i=1, \ldots, \mathrm{n}$.

3. For each individual $i$, obtain the GLS estimator of $\beta_{i}$ by WLS with weights $\hat{\mathrm{w}}_{i j}^{(k)}$. Set $k=k+1$, let $\beta_{i}^{*(k)}, i=1, \ldots, \mathrm{n}$ be these GLS estimators, and return to step 2 .

In step 2, information from all individuals on the (common) intra-individual variance structure is used to estimate $(\sigma, \theta)$ by pooling information across individuals. Since the objective function is simply the sum of the objective functions for all individuals, minimization is no more difficult than for data from a single individual only. See [3] and [4] for details on implementation with standard software. As in the case of individual data, this scheme is iterated a fixed number of times or until convergence.

The final estimates $\beta_{i}^{*}$ from this scheme are proposed in [4] as input to one of the procedures such as GTS for estimation of $\gamma$ and $\Sigma$. These $\beta_{i}^{*}$ are to be preferred to those based on a separate estimate for $\theta$ for each individual because they are likely to be more precise, since they are based on a weighting scheme that takes advantage of the information from all individuals through the "pooled" estimation of $\sigma$ and $\theta$.

To summarize, the procedure is: (1) Obtain $\beta_{i}^{*}$ by the GLS algorithm based on pooled estimation of $(\sigma, \theta)$, and use these estimates to form $V_{i} ;$ (2) Use $\beta_{i}^{*}$ and $V_{i}$ as input to a scheme such as GTS to estimate $(\gamma, \Sigma)$ and obtain refined estimates of $\beta_{i}$ if desired. These "pooled two-stage" (PTS) methods may be used when sufficient data are available to obtain estimates $\beta_{i}^{*}$ from each individual.

\subsection{Methods based on linearization}

The other main class of approaches to estimation in the nonlinear mixed effects 
model is based on linearization of the model (12) by a Taylor series in the random effects $z_{i}$. This idea was first advocated by Beal and Sheiner [7,8]. Under the assumption that $z_{i} \sim(0, \Sigma)$ and $\epsilon_{i} \sim\left(0, I_{m_{i}}\right)$, they proposed that the Taylor series be taken about $z_{i}=0$, leading to the approximate model

$$
\mathrm{y}_{i} \doteq \mathrm{f}_{i}\left\{\mathrm{~h}\left(\mathrm{~W}_{i}, \gamma, 0\right)\right\}+\mathrm{D}_{i}\left(\mathrm{~W}_{i}, \gamma, 0\right) \mathrm{z}_{i}+\sigma \mathrm{G}_{i}\left\{\mathrm{~h}\left(\mathrm{~W}_{i}, \gamma, 0\right), \theta\right\} \epsilon_{i},
$$

where $\mathrm{D}_{i}\left(\mathrm{~W}_{i}, \gamma, \cdot\right)=\mathrm{X}_{i}\left\{\mathrm{~h}\left(\mathrm{~W}_{i}, \gamma, \cdot\right)\right\} \mathrm{H}_{i}\left(\mathrm{~W}_{i}, \gamma, \cdot\right), \mathrm{X}_{i}(\cdot)$ is the $\left(\mathrm{m}_{i} \times \mathrm{p}\right)$ matrix of derivatives of $\mathrm{f}_{i}\left(\beta_{i}\right)$ with respect to $\beta_{i}$, and $\mathrm{H}_{i}\left(\mathrm{~W}_{i}, \gamma, \cdot\right)$ is the $\mathrm{p} \times M$ matrix of partial derivatives of $\mathrm{h}\left(\mathrm{W}_{\boldsymbol{i}}, \gamma, \mathrm{z}_{\boldsymbol{i}}\right)$ with respect to $\mathrm{z}_{\boldsymbol{i}}$. This approximation implies

$$
\mathrm{E}\left(\mathrm{y}_{i}\right) \doteq \mathrm{f}_{i}\left\{\mathrm{~h}\left(\mathrm{~W}_{i}, \gamma, 0\right)\right\} ; \quad \operatorname{Cov}\left(\mathrm{y}_{i}\right) \doteq \mathrm{D}_{i}\left(\mathrm{~W}_{i}, \gamma, 0\right) \Sigma \mathrm{D}_{i}^{\mathrm{T}}\left(\mathrm{W}_{i}, \gamma, 0\right)+\sigma^{2} \mathrm{G}_{i}^{2}\left\{\mathrm{~h}\left(\mathrm{~W}_{i}, \gamma, 0\right), \theta\right\}
$$

If one assumes that the random components $z_{i}$ and $\epsilon_{i}$ are normally distributed, and if (18) is treated as exact, then the $y_{i}$ have $m_{i}$-variate normal distributions with mean and covariance given in (19). This approximation is the basis for the suggestion of Beal and Sheiner to obtain estimates of $(\gamma, \Sigma, \sigma, \theta)$ by maximizing in these parameters the normal likelihood for the data vectors $\mathrm{y}_{i}, i=1, \ldots, \mathrm{n}$, corresponding to these assumptions. In the pharmacokinetics literature, this procedure is referred to as the "First-Order" method, and the estimation scheme corresponding to normal ML is referred to as Extended Least Squares (ELS) [7,8,9]. The method is implemented in the software package NONMEM [14]; the reader is referred to this documentation for computational details. The individual $\beta_{i}$ may be estimated by an empirical Bayes approach as well [14]. NONMEM is widely used in the analysis of population pharmacokinetic data. For some problems, this approach can be computationally intensive.

Recently, Vonesh and Carter [5] have proposed an alternative to the ELS method which is also based on the linearization (18) but is computationally simpler. In [1], their method has been modified to incorporate a variance function $\mathrm{g}$ and estimation of $\theta$. The procedure is a four-step iterative algorithm in the spirit of GLS. The second step is based on the idea that, if $\gamma$ were known in (18), $\left[\mathrm{y}_{i}-\mathrm{f}_{i}\left\{\mathrm{~h}\left(\mathrm{~W}_{i}, \gamma, 0\right)\right\}\right]=$ $\mathrm{D}_{i}\left(\mathrm{~W}_{i}, \gamma, 0\right) z_{i}+\sigma \mathrm{G}_{i}\left\{\mathrm{~h}\left(\mathrm{~W}_{i}, \gamma, 0\right), \theta\right\} \epsilon_{i}$ is a weighted linear "regression model" for individual $i$ with "regression parameter" $z_{i}$, "design matrix" $D_{i}\left(\mathrm{~W}_{i}, \gamma, 0\right)$, and "covariance matrix" $\sigma^{2} \mathrm{G}_{i}^{2}\left\{\mathrm{~h}\left(\mathrm{~W}_{i}, \gamma, 0\right), \theta\right\}$.

Step 1. Let $\mathrm{k}=0$, and obtain the ordinary LS estimator $\hat{\gamma}_{(0)}$ by minimizing in $\gamma$ 


$$
\sum_{i=1}^{n}\left[y_{i}-f_{i}\left\{h\left(W_{i}, \gamma, 0\right)\right\}\right]^{T}\left[y_{i}-f_{i}\left\{h\left(W_{i}, \gamma, 0\right)\right\}\right]
$$

Step 2. Form $\hat{\mathrm{e}}_{i}=\mathrm{y}_{i}-\mathrm{f}_{i}\left\{\mathrm{~h}\left(\mathrm{~W}_{i}, \hat{\gamma}_{(k)}, 0\right)\right\}$, let $\hat{\mathrm{D}}_{i}=\mathrm{D}_{i}\left(\mathrm{~W}_{i}, \hat{\gamma}_{(k)}, 0\right)$, and iterate the following steps:

(i) Let $j=0$. For each individual, obtain an initial estimate of $z_{i}, \hat{z}_{i}^{(j)}=$ $\left(\hat{\mathrm{D}}_{i}^{\mathrm{T}} \hat{\mathrm{D}}_{i}\right)^{-1} \hat{\mathrm{D}}_{i} \hat{\mathrm{e}}_{i}$.

(ii) Form "residuals" $\hat{\mathrm{r}}_{i}=\hat{\mathrm{e}}_{i}-\hat{\mathrm{D}}_{i} \hat{\mathrm{z}}_{i}^{(j)}$ and estimate $(\sigma, \theta)$ by $(\hat{\sigma}, \hat{\theta})$ jointly maximizing

$$
\sum_{i=1}^{n} O_{i}\left\{\hat{r}_{i}, h\left(W_{i}, \hat{\gamma}_{(k)}, 0\right), \sigma, \theta\right)
$$

where $\mathrm{O}_{i}$ is a suitably chosen objective function.

(iii) Form $\hat{\mathrm{G}}_{i}=\mathrm{G}_{i}\left\{\mathrm{~h}\left(\mathrm{~W}_{i}, \hat{\gamma}_{(k)}, 0\right), \hat{\theta}\right\}$, update the random effects estimates for each individual by $\hat{z}_{i}^{(j+1)}=\left(\hat{\mathrm{D}}_{i}^{\mathrm{T}} \hat{\mathrm{G}}_{i}^{-1} \hat{\mathrm{D}}_{i}\right)^{-1} \hat{\mathrm{D}}_{i}^{\mathrm{T}} \hat{\mathrm{G}}_{i}^{-1} \hat{\mathrm{e}}_{i}$, and let $j=j+1$. Return to (ii). Call the final estimates from this process $\hat{z}_{i}^{(k)}, \hat{\sigma}_{(k)}, \hat{\theta}_{(k)}$, and let $\hat{G}_{i}$ $=\mathrm{G}_{i}\left\{\mathrm{~h}\left(\mathrm{~W}_{i}, \hat{\gamma}_{(k)}, 0\right), \hat{\theta}_{(k)}\right\}$

Step 3. Let $S_{z z}=(n-1)^{-1} \sum_{i=1}^{n}\left(\hat{z}_{i}^{(k)}-\bar{z}\right)\left(\hat{z}_{i}^{(k)}-\bar{z}\right)^{T}, \bar{z}=n^{-1} \sum_{i=1}^{n} \hat{z}_{i}^{(k)}$, and estimate $\Sigma$ by $\hat{\Sigma}_{(k)}= \begin{cases}\mathrm{S}_{z z}-\hat{\sigma}_{(k)}^{2} \mathrm{n}^{-1} \sum_{i=1}^{n}\left(\hat{\mathrm{D}}_{i}^{\mathrm{T}} \hat{\mathrm{G}}_{i}^{-1} \hat{\mathrm{D}}_{i}\right)^{-1}, & \hat{\lambda}>\hat{\sigma}_{(k)}^{2} \\ \mathrm{~S}_{z z}-\hat{\lambda}^{2} \mathrm{n}^{-1} \sum_{i=1}^{n}\left(\hat{\mathrm{D}}_{i}^{\mathrm{T}} \hat{\mathrm{G}}_{i}^{-1} \hat{\mathrm{D}}_{i}\right)^{-1}, & \hat{\lambda} \leq \hat{\sigma}_{(k)}^{2}\end{cases}$ where $\hat{\lambda}$ is the smallest root of $\left|S_{z z}-\lambda n^{-1} \sum_{i=1}^{n}\left(\hat{D}_{i}^{T} \hat{G}_{i}^{-1} \hat{D}_{i}\right)^{-1}\right|=0$.

Step 4. Form $\left(\hat{\mathrm{U}}_{i}\right)_{(k)}=\hat{\mathrm{D}}_{i} \hat{\Sigma}_{(k)} \hat{\mathrm{D}}_{i}^{\mathrm{T}}+\hat{\sigma}^{2} \hat{\mathrm{G}}_{i}$ and update estimation of $\hat{\gamma}_{(k+1)}$ by minimizing in $\gamma$

$$
\sum_{i=1}^{n}\left[y_{i}-f_{i}\left\{h\left(V_{i}, \gamma, 0\right)\right\}\right]^{T}\left(\hat{U}_{i}\right)_{(k)}^{-1}\left[y_{i}-f_{i}\left\{h\left(W_{i}, \gamma, 0\right)\right\}\right] .
$$

Let $k=k+1$ and return to Step 2.

If $G_{i} \equiv I_{m_{i}}$ no iteration is required within Step 2. In general, Step 2 is not too intensive since the "regression" fits are linear, and the process often converges after a few iterations. As in Section 4.2, at least one iteration of the entire algorithm should be taken; results often stabilize after three to five iterations. At the end of Step 4, individual estimates may be constructed as $\hat{\beta}_{i}^{(k+1)}=\mathrm{h}\left(\mathrm{W}_{i}, \hat{\gamma}_{(k+1)}, \hat{\mathrm{z}}_{i}^{(k)}\right)$.

Choice of objective function $\mathrm{O}_{i}$ is based on the same ideas as in Section 4.2. Two possibilities are 


$$
\begin{aligned}
\mathrm{PL}_{i}\left(\mathrm{r}_{i}, \mathrm{~h}, \sigma, \theta\right) & =\sum_{j=1}^{m_{i}}\left(\frac{\mathrm{r}_{i}^{2}}{\sigma^{2} \mathrm{~g}^{2}\left\{\mathrm{f}\left(\mathrm{x}_{i j}, \mathrm{~h}\right), \theta\right\}}+\log \left[\sigma^{2} \mathrm{~g}^{2}\left\{\mathrm{f}\left(\mathrm{x}_{i j}, \mathrm{~h}\right), \theta\right\}\right]\right), \\
\mathrm{AR}_{i}\left(\mathrm{r}_{i}, \mathrm{~h}, \sigma, \theta\right) & =\sum_{j=1}^{m_{i}}\left(\frac{\left|\mathrm{r}_{i}\right|}{\sigma \mathrm{g}\left\{\mathrm{f}\left(\mathrm{x}_{i j}, \mathrm{~h}\right), \theta\right\}}+\log \left[\sigma \mathrm{g}\left\{\mathrm{f}\left(\mathrm{x}_{i j}, \mathrm{~h}\right), \theta\right\}\right]\right),
\end{aligned}
$$

Maximization of these objective functions in step 2(ii) can be accomplished using standard software, see $[1,3,4]$.

The covariance matrix of $\hat{\gamma}_{(k+1)}$ obtained at the end of the Step 4 can be estimated by

$$
\hat{\Omega}=\left[\sum_{i=1}^{n}\left\{\mathrm{~W}_{i}^{\mathrm{T}} \hat{\mathrm{X}}_{i}^{\mathrm{T}} \hat{\mathrm{U}}_{i}^{-1} \hat{\mathrm{X}}_{i} \mathrm{~W}_{i}\right\}\right]^{-1}
$$

where $\hat{\mathrm{X}}_{i}$ is $\mathrm{X}_{i}$ evaluated at $\mathrm{h}\left(\mathrm{W}_{i}, \hat{\gamma}_{(k+1)}, 0\right)$ and $\hat{\mathrm{U}}_{i}=$ $\mathrm{D}_{i}\left(\mathrm{~W}_{i}, \hat{\gamma}_{(k+1)}, 0\right) \hat{\Sigma}_{(k)} \mathrm{D}_{i}\left(\mathrm{~W}_{i}, \hat{\gamma}_{(k+1)}, 0\right)^{\mathrm{T}}+\hat{\sigma}_{(k)}^{2} \mathrm{G}_{i}^{2}\left\{\mathrm{~h}\left(\mathrm{~W}_{i}, \hat{\gamma}_{(k+1)}, 0\right), \hat{\theta}_{(k)}\right\}$. This method may be used when information from each individual is sparse as long as $\mathrm{m}_{i} \geq M$.

Lindstrom and Bates [11] suggest that the approximation based on $z_{i} \doteq 0$ can be improved and propose a computational method based on a Taylor series of (12) about $z_{i}$ $=z_{i}^{*}$, for $z_{i}^{*}$ "near" $z_{i}$. This yields the approximate model and moments

$$
\begin{aligned}
\mathrm{y}_{i} \doteq \mathrm{f}_{i}\left\{\mathrm{~h}\left(\mathrm{~W}_{i}, \gamma, \mathrm{z}_{i}^{*}\right)\right\}-\mathrm{D}_{i}\left(\mathrm{~W}_{i}, \gamma, \mathrm{z}_{i}^{*}\right) \mathrm{z}_{i}^{*}+\mathrm{D}_{i}\left(\mathrm{~W}_{i}, \gamma, \mathrm{z}_{i}^{*}\right) \mathrm{z}_{i}+\sigma \mathrm{G}_{i}\left\{\mathrm{~h}\left(\mathrm{~W}_{i}, \gamma, \mathrm{z}_{i}^{*}\right), \theta\right\} \epsilon_{i} ; \\
\quad \mathrm{E}\left(\mathrm{y}_{i}\right) \doteq \mathrm{f}_{i}\left\{\mathrm{~h}\left(\mathrm{~W}_{i}, \gamma, \mathrm{z}_{i}^{*}\right)\right\}-\mathrm{D}_{i}\left(\mathrm{~W}_{i}, \gamma, \mathrm{z}_{i}^{*}\right) \mathrm{z}_{i}^{*} \\
\operatorname{Cov}\left(\mathrm{y}_{i}\right) \doteq \mathrm{D}_{i}\left(\mathrm{~W}_{i}, \gamma, \mathrm{z}_{i}^{*}\right) \Sigma \mathrm{D}_{i}^{\mathrm{T}}\left(\mathrm{W}_{i}, \gamma, \mathrm{z}_{i}^{*}\right)+\sigma^{2} \mathrm{G}_{i}^{2}\left\{\mathrm{~h}\left(\mathrm{~W}_{i}, \gamma, \mathrm{z}_{i}^{*}\right), \theta\right\}
\end{aligned}
$$

To base estimation of $(\gamma, \Sigma, \sigma, \theta)$ on this approximation, it is necessary to have available a value for $z_{i}^{*}$. Lindstrom and Bates propose an iterative scheme in which an estimate of $z_{i}$ is obtained at each iteration. This estimate is inserted in the expressions in (22), and updated estimates of $(\gamma, \Sigma, \sigma, \theta)$ and $z_{i}$ are obtained. The iterative computation is fairly complex; we only summarize the basic idea. At iteration $(k+1)$ :

(1) Given current estimates $\hat{\gamma}_{(k)}, \hat{\Sigma}_{(k)}, \hat{\sigma}_{(k)}, \hat{\theta}_{(k)}$, and $\hat{z}_{i}^{(k)}$, obtain updated estimates $\hat{z}_{i}^{(k+1)}$ of $z_{i}$ : maximize the likelihood for $z_{i}$ given the data $y_{i j}$ under the assumption that the $z_{i}$ and $\epsilon_{i}$ are normal, obtaining the estimates

$$
\begin{aligned}
\hat{\mathrm{z}}_{i}^{(k+1)}= & {\left[\hat{\sigma}_{(k)}^{-2} \hat{\mathrm{D}}_{i}\left\{\hat{\mathrm{G}}_{i}^{2}\right\}^{-1} \hat{\mathrm{D}}_{i}+\hat{\Sigma}_{(k)}\right]^{-1} \times } \\
& \left(\hat{\sigma}_{(k)}^{-2} \hat{\mathrm{D}}_{i}\left\{\hat{\mathrm{G}}_{i}^{2}\right\}^{-1}\left[\mathrm{y}_{i}-\mathrm{f}_{i}\left\{\mathrm{~h}\left(\mathrm{~W}_{i}, \hat{\gamma}_{(k)}, \hat{\mathrm{z}}_{i}^{(k)}\right)\right\}+\hat{\mathrm{D}}_{i} \hat{\mathrm{z}}_{i}^{(k)}\right),\right.
\end{aligned}
$$

where $\hat{\mathrm{D}}_{i}=\mathrm{D}_{i}\left(\mathrm{~W}_{i}, \hat{\gamma}_{(k)}, \hat{z}_{i}^{(k)}\right)$ and $\hat{\mathrm{G}}_{i}=\mathrm{G}_{i}\left\{\mathrm{~h}\left(\mathrm{~W}_{i}, \hat{\gamma}_{(k)}, \hat{z}_{i}^{(k)}\right), \hat{\theta}_{(k)}\right\}$.

(2) Redefine $\hat{\mathrm{D}}_{i}=\mathrm{D}_{i}\left(\mathrm{~W}_{i}, \hat{\gamma}_{(k)}, \hat{\mathrm{z}}_{i}^{(k+1)}\right)$ and $\hat{\mathrm{G}}_{i}=\mathrm{G}_{i}\left\{\mathrm{~h}\left(\mathrm{~W}_{i}, \hat{\gamma}_{(k)}, \hat{\mathrm{z}}_{i}^{(k+1)}\right), \hat{\theta}_{(k)}\right\}$. Obtain 
estimates $\hat{\gamma}_{(k+1)}, \hat{\Sigma}_{(k+1)}, \hat{\sigma}_{(k+1)}, \hat{\theta}_{(k+1)}$ maximizing in $(\gamma, \Sigma, \sigma, \theta)$ the normal likelihood for the data vectors $\mathrm{y}_{i}, i=1, \ldots, \mathrm{n}$, with means and covariance matrices

$$
\mathrm{f}_{i}\left\{\mathrm{~h}\left(\mathrm{~W}_{i}, \gamma, \hat{\mathrm{z}}_{i}^{(k+1)}\right)\right\}-\hat{\mathrm{D}}_{i} \hat{z}_{i}^{(k+1)}, \quad \hat{\mathrm{D}}_{i} \Sigma \hat{\mathrm{D}}_{i}^{\mathrm{T}}+\sigma^{2} \mathrm{G}_{i}^{2}\left\{\mathrm{~h}\left(\mathrm{~W}_{i}, \hat{\gamma}_{(k)}, \hat{\mathrm{z}}_{i}^{(k+1)}\right), \theta\right\}
$$

respectively.

We refer the reader to [11] for full details and computational strategies. In the above, we have modified the Lindstrom and Bates proposal for nonlinear $h$ and to incorporate estimation of $\theta$ in a variance function depending on $\beta_{i}$. At the end of (2), estimates of the individual $\beta_{i}$ may be obtained as $\hat{\beta}_{i}^{(k+1)}=\mathrm{h}\left(\mathrm{W}_{i}, \hat{\gamma}_{(k+1)}, \hat{\mathrm{z}}_{i}^{(k+1)}\right)$.

\subsection{Other methods}

As pointed out in Section 4.1, the major classes of methods that we have discussed are based on approximations or individual estimates because the complexity of the model (12) makes the use of standard methodology difficult. This is because it is not possible in general to specify a distribution for the data vectors $y_{i}$, even with distributional assumptions for $z_{i}$ and $\epsilon_{i}$. We now examine this problem more closely. If we were to assume, for example, that the $\epsilon_{i}$ are normally distributed, this would imply that, given $\beta_{i}$ (and hence, given $z_{i}$ ), $y_{i}$ are normally distributed with mean vector $f_{i}\left(\beta_{i}\right)$ and covariance matrix $\sigma^{2} G_{i}^{2}\left(\beta_{i}, \theta\right)$. Writing $\phi(a, B)$ to be the normal density with mean vector a and covariance matrix $\mathrm{B}$, if $\mathrm{z}_{i}$ is assumed to be distributed with some density $\psi(\cdot \mid \Sigma)$ depending on $\Sigma$, the exact likelihood for the response vector $y_{i}$ is

$$
\int \phi\left(\mathrm{f}_{i}\left\{\mathrm{~h}\left(\mathrm{~W}_{i}, \gamma, \mathrm{z}\right)\right\}, \sigma^{2} \mathrm{G}_{i}^{2}\left\{\mathrm{~h}\left(\mathrm{~W}_{i}, \gamma, \mathrm{z}\right), \theta\right\}\right) \psi(\mathrm{z} \mid \Sigma) \mathrm{dz}
$$

the likelihood for the entire data set would be the product of $\mathrm{n}$ such integrals, one for each individual. Even if $\psi$ is the normal density $\phi(0, \Sigma)$, this integral is not analytically tractable for general functions $f, g$, and $h$.

Recently, methods have been proposed which, under various assumptions, represent attempts to perform this integration in order to estimate the parameters. A full description of these methods is beyond the scope of our discussion; we provide only a brief summary and references.

Gelfand, et al. [15] take a full Bayesian approach to the nonlinear mixed effects model. They assume that $z_{i}$ and $\epsilon_{i}$ are normally distributed random vectors and place prior distributions on the parameters $\gamma, \Sigma$, and $\sigma$. (They do not consider estimation of parameters $\theta$ in a variance function $g$, although their idea can be extended to this case.) 
In this framework, several integrations, including that in (23), need to be performed. A computationally intensive Gibbs sampling algorithm is advocated to perform these integrations; a description of this approach and specific details are given in [15].

Mallet et al. [16] note that the assumption that $z_{i}$ are normally distributed may be questionable in population pharmacokinetic analysis. They propose estimation of the density of $\mathrm{z}_{i}, \psi$, in (23) by a nonparametric method. The parameters $\gamma$ and $\sigma$ are then estimated by integrating (23) with this estimate inserted for $\psi$. Since the estimate for $\psi$ consists of a finite number of points, the integral becomes a sum, which may be evaluated easily. See [16] for a full exposition.

Davidian and Gallant [17] also eschew the standard normality assumption for $\psi$. They propose a different nonparametric estimator for $\psi$ which is not a finite number of points but rather is a smooth function of z. By using numerical integration, they compute the integral (23) and estimate $\psi, \gamma, \Sigma, \sigma$, and $\theta$ simultaneously. The form of the estimator for $\psi$ includes $\phi$, the normal density, as a special case.

The methods in this section can involve fairly substantial computational costs. For all methods, including those discussed in Sections 4.2 and 4.3, very little is known about relative performance. This issue is a basis for current research.

\section{EXAMPLES REVISITED}

\subsection{Bioassay of relaxin by RIA}

Calibration of unknown samples for the current run of a cell-based bioassay such as that for relaxin is always based on the standard curve for that run. As noted in Section 2.1 , accurate characterization of intra-assay variability in the response is essential for assessing the precision of calibration. We now illustrate how this issue may be addressed for the relaxin bioassay within the nonlinear mixed effects model framework.

Variability in assay responses is an increasing function of response level, and a standard model for characterizing this feature is the power of the mean variance function [2]. That is, for run $i$,

$$
\mathrm{g}\left\{\mathrm{f}\left(\mathrm{x}_{i j}, \beta_{i}\right), \theta\right\}=\mathrm{f}\left(\mathrm{x}_{i j}, \beta_{i}\right)^{\theta}
$$

for some power $\theta$. It is commonly acknowledged that for many assays, usual default choices for this power, such as $\theta=0.5$ or 1.0 , may be inappropriate [18]. Thus, one objective of an analysis is to determine an appropriate value for $\theta$ for the $i$ th run. The choice of $\theta$ often has little impact on the estimated values of the parameters of the fourparameter logistic model (1) obtained by a weighted regression fit and on subsequent 
calibrated values. However, the value of $\theta$ used directly affects one's ability to assess the precision of calibration accurately, as the following development shows.

A standard technique for evaluating intra-assay precision associated with calibration of an unknown sample for a given run is to construct a precision profile [19]. Such a profile is a plot of estimated precision of an estimated concentration against concentration across the assay range. To illustrate one way to construct a precision profile, let $d$ be the inverse of the four-parameter logistic function, that is, $x=d(y, \beta)=$ $\exp \left[\beta_{3}+\log \left\{\left(\beta_{2}-\mathrm{y}\right) /\left(\mathrm{y}-\beta_{1}\right)\right\} / \beta_{4}\right]$, and let $\mathrm{d}_{\mathrm{y}}(\mathrm{y}, \beta)$ and the $\mathrm{p}$-variate vector-valued function $\mathrm{d}_{\beta}(\mathrm{y}, \beta)$ represent the derivatives of $\mathrm{d}$ with respect to $\mathrm{y}$ and $\beta$, respectively. Then if $y_{0}$ is the average response for $r$ replicates of an unknown sample for the $i$ th run at concentration $\mathrm{x}_{0}$, the estimated value of $\mathrm{x}_{0}$ is given by $\hat{\mathrm{x}}_{0}=\mathrm{d}\left(\mathrm{y}_{0}, \hat{\beta}_{i}\right)$, where $\hat{\beta}_{i}$ is an estimate of $\beta_{i}$ with estimated covariance matrix $V_{i}$. An estimated approximate variance for $\hat{x}_{0}$ may be obtained by a Taylor series:

$$
\operatorname{Var}\left(\hat{\mathrm{x}}_{0}\right)=\mathrm{d}_{y}^{2}\left(\mathrm{y}_{0}, \hat{\beta}_{i}\right) \hat{\sigma}^{2} \mathrm{~g}^{2}\left(\mathrm{y}_{0}, \hat{\theta}\right) / \mathrm{r}+\mathrm{d}_{\beta}^{\mathrm{T}}\left(\mathrm{y}_{0}, \hat{\beta}_{i}\right) \mathrm{V}_{i} \mathrm{~d}_{\beta}\left(\mathrm{y}_{0}, \hat{\beta}_{i}\right)
$$

where $(\hat{\sigma}, \hat{\theta})$ are estimates of the intra-assay variance parameters. The first term in (25) reflects uncertainty in the measurement $y_{0}$ and will usually dominate the second term, which corresponds to uncertainty in the fitted standard curve. A precision profile is constructed as $\left\{\operatorname{Var}\left(\hat{\mathrm{x}}_{0}\right)\right\}^{1 / 2} / \hat{\mathrm{x}}_{0}$ versus $\hat{\mathrm{x}}_{0}$. Since the first term in (25) depends on $(\sigma, \theta)$, assessment of precision of calibration will be sensitive to the values of $\sigma$ and $\theta$ used.

Appropriate values for $(\sigma, \theta)$ in this context are often determined by estimation from the available data, usually based on data from the current run only, using, for example, the GLS algorithm for individual data given in Section 4.2. Although sufficient data are available to provide reliable estimates of $\beta_{i}$ based on data from a single run, estimates of $(\sigma, \theta)$ obtained in this way are usually less reliable, mainly because estimation of variance parameters is more difficult.

This difficulty may be avoided if one is willing to assume a similar pattern of intraassay varability across runs. Although the values of $(\sigma, \theta)$ may be expected to vary from run to run during assay development, once assay procedures have stabilized, supposition of common values of $\sigma$ and $\theta$ across assay runs is not unreasonable. The nonlinear mixed effects model (12) provides a framework for analysis under this assumption, with $\mathrm{f}$ as the four-parameter logistic function, $\mathrm{g}$ the power of the mean model (24), and $h$ as in (5). The GLS algorithm in Section 4.2 where data are pooled across all available runs is a natural way to estimate the common $(\sigma, \theta)$. 
Under these assumptions, Table 4 displays the results of several fits to the relaxin data, both separately by assay run and pooled across assay runs. Because results were very similar for both objective functions in (17) for individual and pooled estimation, we report results for PL only. Although individual estimates of $\theta$ are fairly similar across runs, the value for run 9 seems somewhat aberrant. This is probably attibutable to the difficulty in estimating variance parameters mentioned above. The pooled estimate for $\theta$ based on data from all nine runs indicates that a constant CV model for intra-assay variance would be a reasonable choice for routine use.

In Figure 5, a precision profile for a single replicate of an unknown sample is constructed based on each fit for run 9. The profile based on the LS fit is inappropriate, since it takes no account of the nature of intra-assay variance. The profiles based on individual and pooled GLS fits differ appreciably over a large range of concentrations, reflecting the sensitivity of calibration inference to the method used to characterize intra-assay variability. If the model assumptions are correct, then the profile based on the pooled GLS fit will provide the most accurate assessment of precision.

More extensive analysis of this example and further discussion of issues arising in calibration, including the use of refined estimates of $\beta_{i}$ from GTS as a basis for calibration, is given in [18].

\subsection{Water transport kinetics of high flux hemodialyzers}

An objective of the dialyzer study was to compare the kinetic properties of dialyzers operated at the two flow rates. We now address this question in the context of the nonlinear mixed model framework, assuming the kinetic model $f$ in (3) throughout.

In a preliminary analysis, Vonesh and Carter [5] determined that $\beta_{3}$, transmembrane pressure, was constant across dialyzers within each flow rate, although the value of $\beta_{3}$ was likely different for the two flow rates. Thus, we follow Vonesh and Carter and adopt the following inter-individual regression model $h$ for the vector of kinetic parameters $\beta_{i}=\left[\beta_{i 1}, \beta_{i 2}, \beta_{i 3}\right]^{\mathrm{T}}$ for individual $i$. For dialyzer from flow rate $l, l=200$, 300:

$$
\beta_{i 1}=\gamma_{1 l}+z_{i 1}, \quad \beta_{i 2}=\gamma_{2 l}+z_{i 2}, \quad \beta_{i 3}=\gamma_{3 l},
$$

where $\gamma_{l}=\left[\gamma_{1 l}, \gamma_{2 l}, \gamma_{3 l}\right]^{\mathrm{T}}$ is the vector of "typical" kinetic parameters for a dialyzer operated at flow rate $l=200$ or $300 \mathrm{ml} / \mathrm{min}$, and $z_{i}=\left[z_{i 1}, z_{i 2}\right]^{\mathrm{T}}$ is the random vector describing how the first two parameters, maximum ultrafiltration rate and permeability transport rate, vary in both flow rate populations. This model may be written as $\beta_{i}=$ $\mathrm{W}_{i} \gamma+\mathrm{R}_{i} \mathrm{z}_{i}$, where $\gamma=\left[\gamma_{200}^{\mathrm{T}}, \gamma_{300}^{\mathrm{T}}\right]^{\mathrm{T}}(6 \times 1), \mathrm{W}_{i}$ is defined in $(8)$, and $\mathrm{R}_{i}$ is the $(3 \times 2)$ 
matrix whose columns are the first two columns of $I_{3}$.

From Figure 3, intra-dialyzer variability is an increasing function of ultrafiltration level. To accommodate this feature, we assume that intra-dialyzer variance follows the power of the mean model (24). In their analysis, Vonesh and Carter [5] assumed constant intra-dialyzer variance.

The two-stage methods in Section 4.2 do not allow for the possibility that some components of $\beta_{i}$ are fixed; the methods based on linearization in Section 4.3 do accommodate such a model. We thus used the four-step GLS algorithm given in Section 4.3 to estimate $(\gamma, \Sigma, \sigma, \theta)$. Results were similar for the two objective functions in (20); hence, we report those for PL only. The process stabilized after three iterations in that the relative change in parameter estimates between successive iterations was less than $10^{-4}$. The estimates are:

$$
\begin{gathered}
\hat{\gamma}=[4512.782,0.021,21.922,6253.007,0.013,21.791]^{\mathrm{T}}, \\
\Sigma=\left[\begin{array}{cc}
131142.26 & -0.325 \\
-0.325 & 7.53 \times 10^{-6}
\end{array}\right]
\end{gathered}
$$

$(\hat{\sigma}, \hat{\theta})=(30.04,0.26)$. The estimated value of the power parameter $\theta$ suggests that variance in measurements on a given dialyzer increases like the square root of mean ultrafiltration rate.

Standard errors for $\hat{\gamma}$ may be obtained as the square roots of the estimated covariance matrix $\hat{\Omega}$ in (21). For the assumed model, $\hat{\Omega}$ is a $(6 \times 6)$ block diagonal matrix with $(3 \times 3)$ blocks

$$
\begin{aligned}
& \hat{\Omega}_{200}=\left[\begin{array}{ccc}
15776.794 & -6.715 \times 10^{-2} & -5.500 \\
\cdot & 1.776 \times 10^{-6} & 2.665 \times 10^{-4} \\
\cdot & \cdot & 0.2688
\end{array}\right], \\
& \hat{\Omega}_{300}=\left[\begin{array}{ccc}
20668.417 & -7.289 \times 10^{-2} & -18.568 \\
\cdot & 1.053 \times 10^{-6} & 1.941 \times 10^{-4} \\
\cdot & \cdot & 0.4175
\end{array}\right],
\end{aligned}
$$

corresponding to the $(3 \times 1)$ components $\hat{\gamma}_{200}$ and $\hat{\gamma}_{300}$ of $\hat{\gamma}$, respectively.

An objective of this study was to determine whether kinetic properties of dialyzers differ between the two flow rates. A formal statistical hypothesis test of this issue is the three degree of freedom test of the hypotheses 
$\mathrm{H}_{0}: \gamma_{1,200}-\gamma_{1,300}=0, \gamma_{2,200}-\gamma_{2,300}=0 \gamma_{3,200}-\gamma_{3,300}=0 \quad$ vs. $\mathrm{H}_{1}: \mathrm{H}_{0}$ not true,

which contrasts the three kinetic parameters for the two flow rates. $\mathrm{H}_{0}$ may be written in the form of a general linear hypothesis as $\mathrm{H}_{0}: \mathrm{L} \gamma=0$ vs. $\mathrm{H}_{1}: \mathrm{L} \gamma \neq 0$, where $\mathrm{L}=$ $\left[I_{3} \mid-I_{3}\right]$. By standard statistical theory $[1,5]$, the test may be conducted by comparing the test statistic

$$
\mathrm{T}^{2}=\hat{\gamma}^{\mathrm{T}} \mathrm{L}^{\mathrm{T}}\left(\mathrm{L} \hat{\Omega} \mathrm{L}^{\mathrm{T}}\right)^{-1} \mathrm{~L} \hat{\gamma}
$$

to critical values from the chi-square distribution with $\nu$ degrees of freedom, where $\nu$ is the degrees of freedom associated with the test (equivalently, the number of rows of $\mathrm{L}$ ). For the GTS method of Section 4.2, an analogous test statistic is $\mathrm{T}_{*}^{2}=$ $\hat{\gamma}^{\mathrm{T}} \mathrm{L}^{\mathrm{T}}\left(\mathrm{L} \hat{\Delta} \mathrm{L}^{\mathrm{T}}\right)^{-1} \mathrm{~L} \hat{\gamma}$, where $\hat{\gamma}$ is the final estimate of $\gamma$ obtained from the GTS algorithm at convergence and $\hat{\Delta}$ is given in (16).

For the dialyzer data, $T^{2}=86.778$. From a table of chi-square critical values with 3 degrees of freedom, this is highly significant $(p<0.0001)$, suggesting that, overall, kinetic properties of dialyzers operated at $200 \mathrm{ml} / \mathrm{min}$ differ from those of dialyzers operated at $300 \mathrm{ml} / \mathrm{min}$. Inspection of the estimate $\hat{\gamma}$ indicates that this result is largely due to differences in the first two components of $\gamma_{200}$ and $\gamma_{300}$, maximum attainable ultrafiltration rate and hydraulic permeability transport rate, for the two flow rates. This is the same qualitative result obtained in [5] assuming constant intra-dialyzer variance. Although our conclusion is the same, the knowledge that intra-dialyzer variance increases with mean response is useful for further study of the process. Evidence suggesting that incorrect specification of the intra-individual variance function can produce biased, less reliable estimates of $\gamma$ and $\Sigma$ is given in $[1,4]$.

\subsection{Pharmacokinetics of cefamandole}

One objective of a pilot pharmacokinetic study is to characterize intra-subject variation, which is typically an increasing function of plasma level. A second objective is to determine a suitable kinetic model and to obtain preliminary estimates of the values of the pharmacokinetic parameters. All of this information is used in subsequent design and analysis of pharmacokinetic studies in a patient population. These objectives may be addressed within the nonlinear mixed effects model framework.

From Section 2.3, the biexponential model is a suitable representation of the kinetics of cefamandole at time $x$. This model is often written in the form

$$
f(x, \beta)=e^{\beta_{1}} \exp \left(e^{-\beta_{2}} x\right)+e^{\beta_{3}} \exp \left(e^{-\beta_{4}} x\right)
$$


in order to ensure positivity of the parameter estimates [20]. We adopt this model for $f$ in our analysis of the cefamandole data.

A standard model for intra-subject variance in pharmacokinetic applications is the power of the mean model (24). As discussed by [9], for pharmacokinetic data, usual default values for $\theta$ are often inappropriate, so it is necessary to estimate the power parameter $\theta$ from the data. Since the same assay is used to process blood samples from all subjects, it is not unreasonable to expect a similar pattern of intra-subject variability for all subjects. Thus, we assume that the intra-subject variance function $g$ is given by (24) with unknown power $\theta$, common to all individuals, to be estimated.

Because no information on individual attributes is available, we take the interindividual regression model $\mathrm{h}$ to be

$$
\beta_{i}=\gamma+z_{i}
$$

where $\beta_{i}$ is the $(4 \times 1)$ vector of parameters in the order given in (25) for the $i$ th subject. Within this framework, $\gamma$ is the vector of "typical" pharmacokinetic parameters. Estimates of $\gamma$ and $\Sigma$, the covariance matrix of $z_{i}$, provide the required preliminary information on kinetics as well as a sense of how kinetic properties vary. Variability in the kinetic parameters is usually expressed as a coefficient of variation, that is, the square root of the appropriate diagonal element of $\hat{\Sigma}$ (the estimate of standard deviation for the kinetic parameter) divided by the corresponding component of $\hat{\gamma}$, times $100 \%$.

Under these assumptions, we used the GLS algorithm of Section 4.2 where the data are pooled across subjects to estimate $(\sigma, \theta)$ using the PL objective function. Final estimates $\beta_{i}^{*}$ and corresponding estimated covariance matrices $\mathrm{V}_{i}$ from this procedure, were input to the GTS algorithm to estimate $\gamma$ and $\Sigma$. Refined estimates $\hat{\beta}_{i}$ of the individual parameters $\beta_{i}$ were also obtained. Results are summarized in Table 5(a). Refined estimates of the elements of $\beta_{i}$ differ from the pooled GLS estimates; this is due to the well-known phenomenon that empirical Bayes estimates borrow information across the sample to "shrink" individual estimates toward the mean parameter value.

The four-step GLS algorithm in Section 4.3 was also used to estimate $(\gamma, \Sigma, \sigma, \theta)$ and obtain individual estimates $\hat{\hat{\beta}}_{i}=\hat{\gamma}+\hat{z}_{i}$, where $\hat{\gamma}$ and $\hat{z}_{i}$ are the final estimates of $\gamma$ and $z_{i}$. The PL objective function was used for estimation of $(\sigma, \theta)$. Results after 3 iterations of the algorithm are given in Table 5(b). Estimated CVs for the parameters based on both analyses suggest that rate constants (and hence, half life) vary appreciably among subjects, although this result is to be interpreted with care, as we now discuss. 
One would hope that the two different estimation methods would produce comparable results, and they do yield similar estimates for $\gamma, \sigma$, and $\theta$. The most striking difference between the two analyses is that the estimates of the covariance matrix $\Sigma$ differ appreciably. This disagreement reflects the idea that estimation of variation in the population is unlikely to be very reliable when based on such a small sample from the population (six subjects). In general, estimation of $\Sigma$ should not be attempted unless the number of individuals sampled (n) is large. The quality of estimates of $\gamma, \sigma$, and $\theta$ are not as adversely affected by small $n$, in our experience.

\section{CONCLUSION}

The nonlinear mixed effects model is a useful and flexible framework for the analysis of repeated measurement data. In this tutorial, we have reviewed several approaches to estimation and inference within this framework and illustrated these with data sets from a range of areas of application. The examples illustrate the types of analyses that are possible as well as the care that must be taken in interpreting the results. In particular, the cefamandole example highlights the fact that estimation of variation in the population of individuals can be unreliable when the number of individuals sampled is small. Research is currently under way to investigate this and other issues.

The cefamandole example illustrates the analysis of pharmacokinetic data from a small, controlled pilot study. Clinical data from a patient population usually consist of only a few plasma concentration measurements on each of a large number of subjects along with information on patient attributes such as physical characteristics and disease status. The analysis of these data is often complex, and the improvement of existing techniques as well as the development of new procedures is an area of current research $[21,22]$. An extensive bibliography of references on population pharmacokinetic analyses is given in [23].

\section{REFERENCES}

1 M. Davidian and D. Giltinan, Some estimation methods for nonlinear mixed effects models, Journal of Biopharmaceutical Statistics, 1 (1992) in press.

2 D. Rodbard, R.H. Lenox, H.L. Wray and D. Ramseth, Statistical characterization of the random errors in the radioimmunoassay dose-response variable, Clinical Chemistry, 22 (1976) $350-358$

3 M. Davidian and P.D. Haaland, Regression and calibration with nonconstant error variance, Chemometrics and Intelligent Laboratory Systems, 9 (1990) $231-248$.

4 M. Davidian and D.M. Giltinan, Some simple methods for estimating intraindividual variability in nonlinear mixed effects models, Biometrics, (1992) in press.

5 E.F. Vonesh and R.L. Carter, Mixed effects nonlinear regression for unbalanced repeated measures, Biometrics, 48 (1992) $1-18$. 
6 N.S. Aziz, J.G. Gambertoglio, E.T. Lin, H. Grausz and L.Z. Benet, Pharmacokinetics of cefamandole using a HPLC assay, Journal of Pharmacokinetics and Biopharmaceutics, 6 (1978) $153-164$.

7 S.L. Beal and L.B. Sheiner, Estimating population kinetics, CRC Critical Reviews in Biomedical Engineering, 8 (1982) 195 - 222.

8 S.L. Beal and L.B. Sheiner, Methodology of population pharmacokinetics, Drug Fate and Metabolism-Methods and Techniques, Eds. E.R. Garrett and J.L. Hirtz, Marcel Dekker, New York, 1985.

9 S.L. Beal and L.B. Sheiner, Heteroscedastic nonlinear regression, Technometrics, 30 (1988) $327-338$.

10 G.A.F. Seber and C.J. Wild, Nonlinear Regression, Wiley, New York, 1989.

11 M.J. Lindstrom and D.M. Bates, Nonlinear mixed effects models for repeated measures data, Biometrics, 46 (1990) $673-687$.

12 J.L. Steimer, A. Mallet, J.L. Golmard and J.F. Boisvieux, Alternative approaches to estimation of population pharmacokinetic parameters: comparison with the nonlinear mixed effect model, Drug Metabolism Reviews, 15 (1984) $265-292$.

13 A. Racine-Poon, A Bayesian approach to nonlinear random effects models, Biometrics, 41 (1985) $1015-1023$.

14 A.J. Boeckmann, L.B. Sheiner and S.L. Beal, NONMEM User's Guide, Parts I-IV, University of California at San Francisco, 1990.

15 A.E. Gelfand, S.E. Hills, A. Racine-Poon and A.F.M. Smith, Illustration of Bayesian inference in normal data models using Gibbs sampling, Journal of the American Statistical Association, 85 (1990) $972-985$.

16 A. Mallet, F. Mentré, J-L Steimer, and F. Lokiec, Nonparametric maximum likelihood estimation for population pharmacokinetics, with application to cyclosporine, Journal of Pharmacokinetics and Biopharmaceutics, 16 (1988) $311-327$.

17 M. Davidian and A.R. Gallant, The nonlinear mixed effects model with a smooth random effects density, Biometrika, in revision.

18 D.M. Giltinan and M. Davidian, Assays for recombinant proteins: a problem in nonlinear calibration, invited submission, Case Studies in Biometry, Wiley, New York, 1992.

19 R.P. Ekins and P.R. Edwards, The precision profile: its use in assay design, assessment, and quality control, Immunoassays for Clinical Chemistry, Eds. W.M. Hunter and J.E.T. Corrie, Churchill Livingston, Edinburgh, 1983.

20 L.B. Sheiner and S.L. Beal, Evaluation of methods for estimating population pharmacokinetic parameters. III. Monoexponential model: routine clinical pharmacokinetic data, Journal of Pharmacokinetics and Biopharmaceutics, 11 (1983) $303-319$.

21 J.W. Mandema, D. Verotta and L.B. Sheiner, Building population pharmacokineticpharmacodynamic models, submitted to Journal of Pharmacokinetics and Biopharmaceutics.

22 M. Davidian and A.R. Gallant, Smooth nonparametric maximum likelihood estimation for population pharmacokinetics, with application to quinidine, Journal of Pharmacokinetics and Biopharmaceutics in press.

23 L.B. Sheiner and T.M. Ludden, Population pharmacokinetics/dynamics, Annual Review of Pharmacological Toxicology, 32 (1992) $185-209$. 
Figure Captions

Fig. 1. Concentration-response data for two runs of the relaxin bioassay.

Fig. 2. Ultrafiltration rate data for twenty high flux hemodialyzers operated at two blood flow rates. Each symbol represents data for a different dialyzer.

Fig. 3. Standardized LS residuals versus predicted response for the dialyzer data. Each symbol represents data for a different dialyzer.

Fig. 4. Cefamandole plasma level-time profiles for six subjects. Each symbol represents data for a different subject.

Fig. 5. Precision profiles for three different fits to run 9 of the relaxin bioassay: dotted line, LS based on individual data; dashed line, GLS-PL based on individual data; solid line, GLS-PL based on pooled data. 


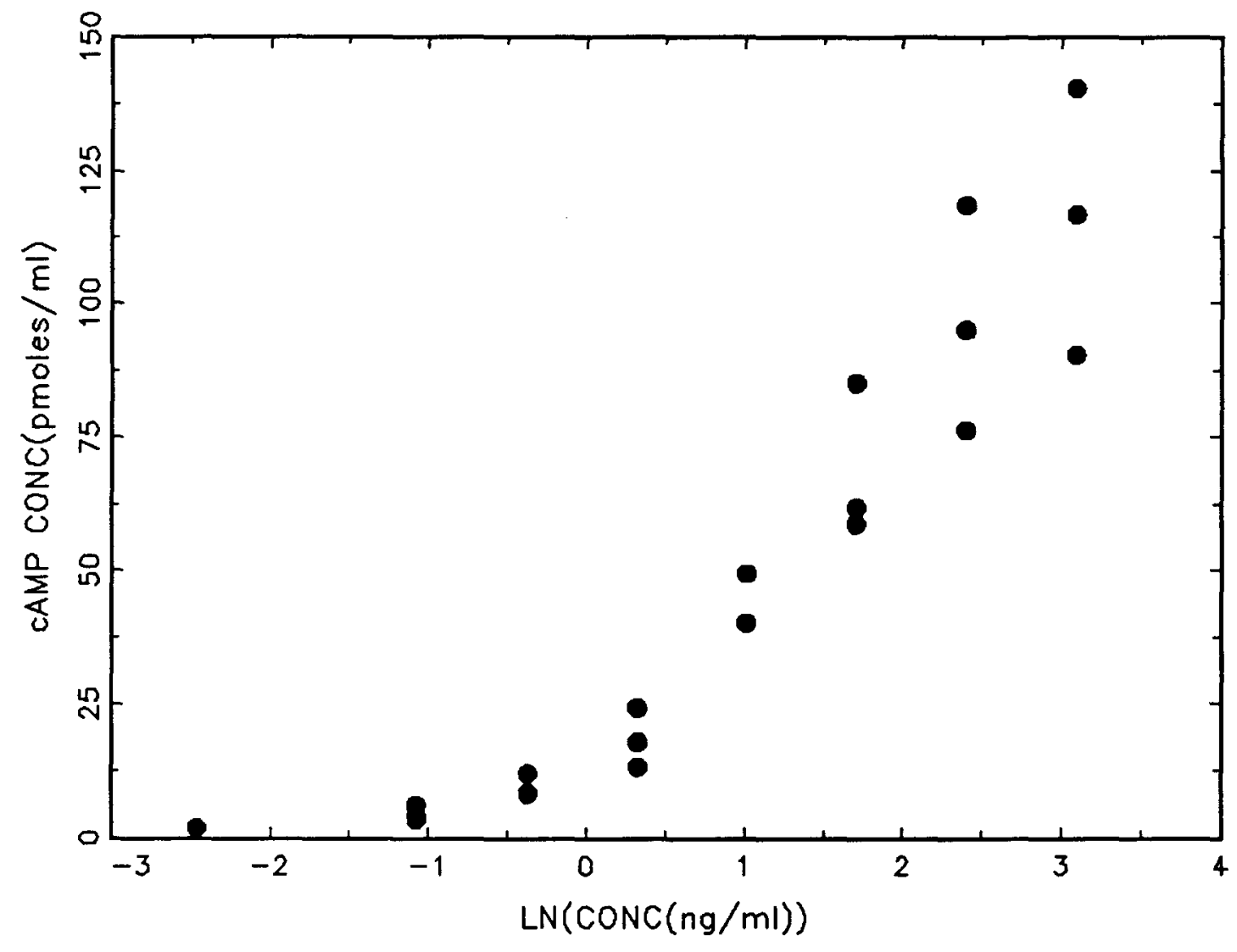

Fig. 1 . 


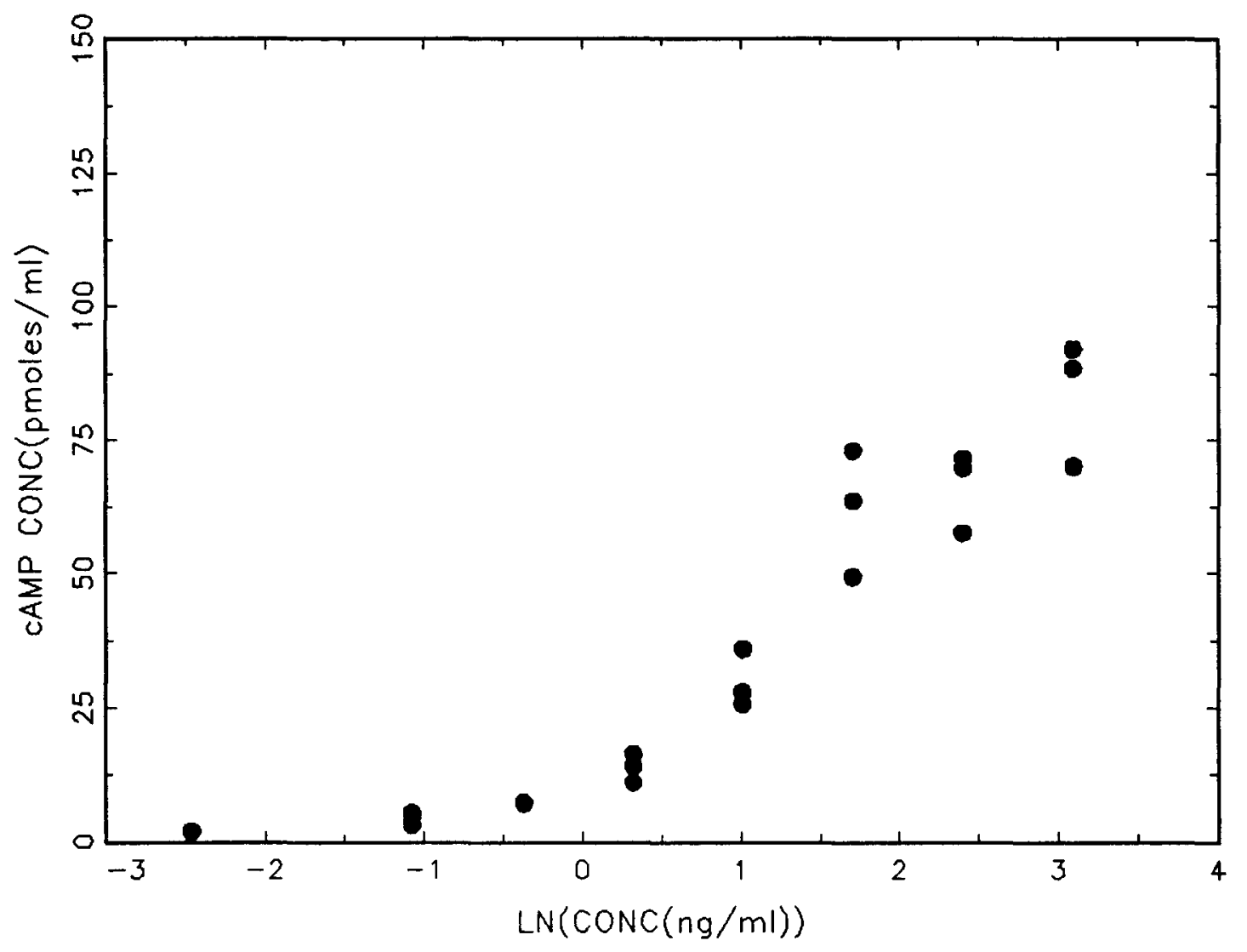


FLOW RATE $=200 \mathrm{ml} / \mathrm{min}$

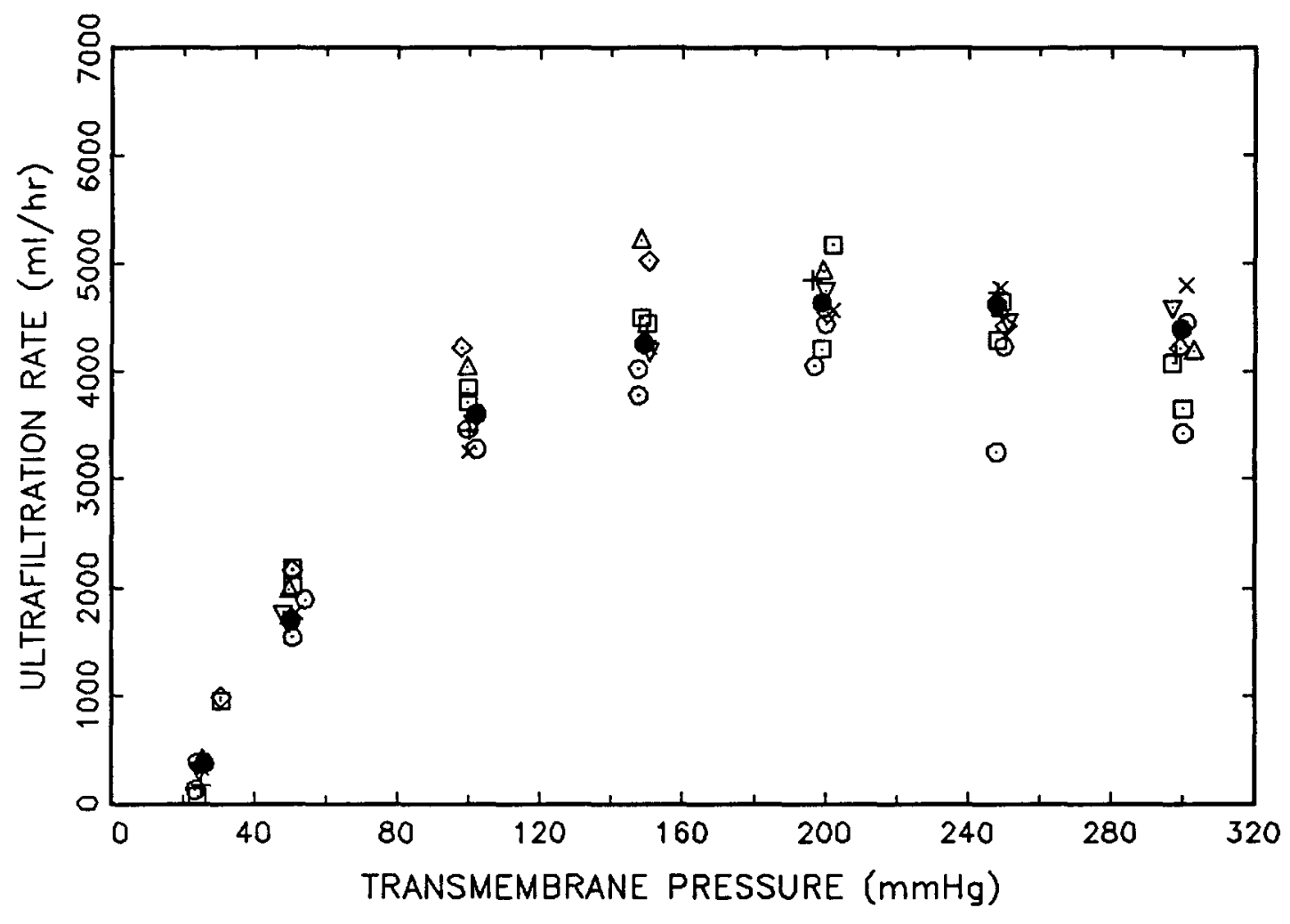

Fig. 2 . 


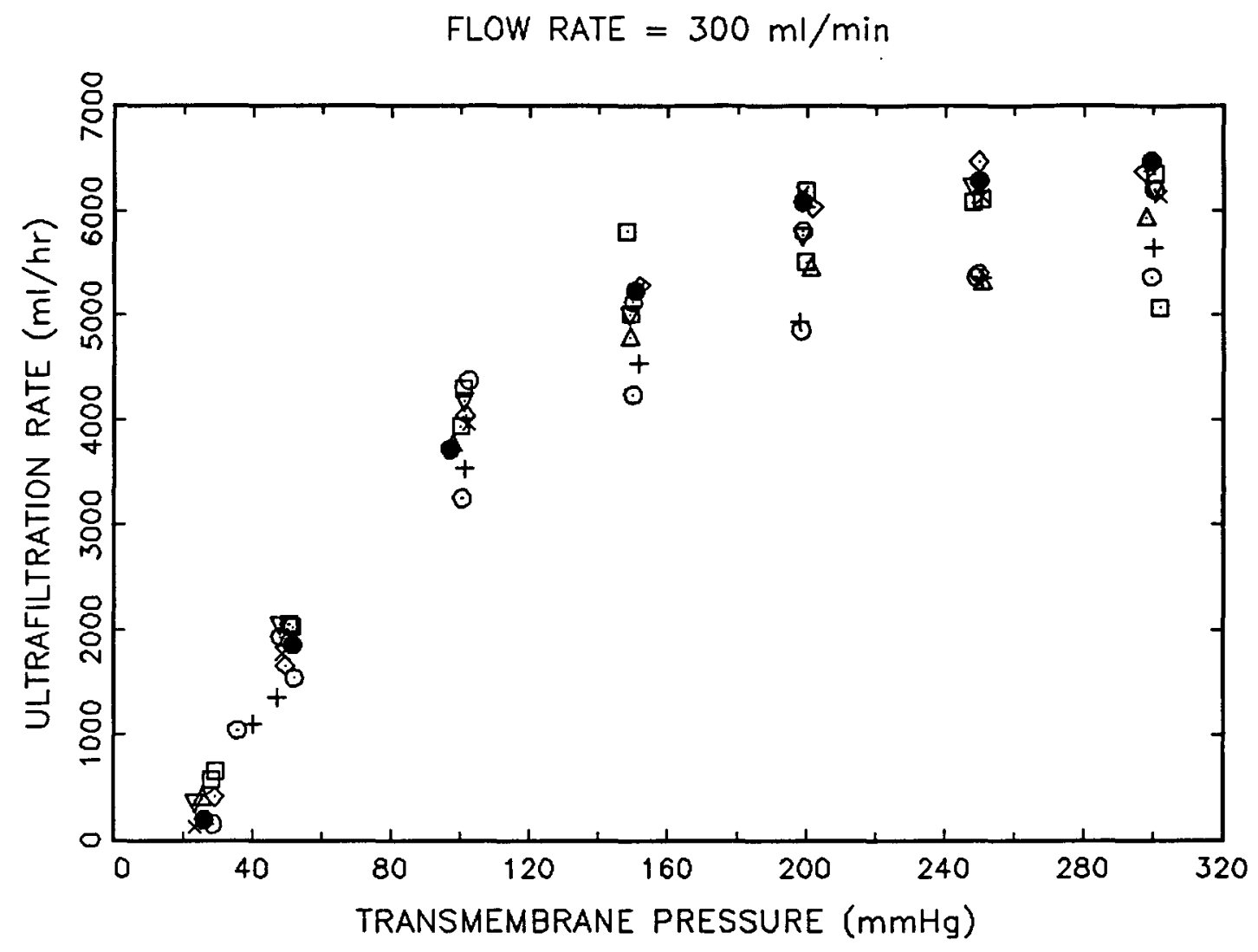




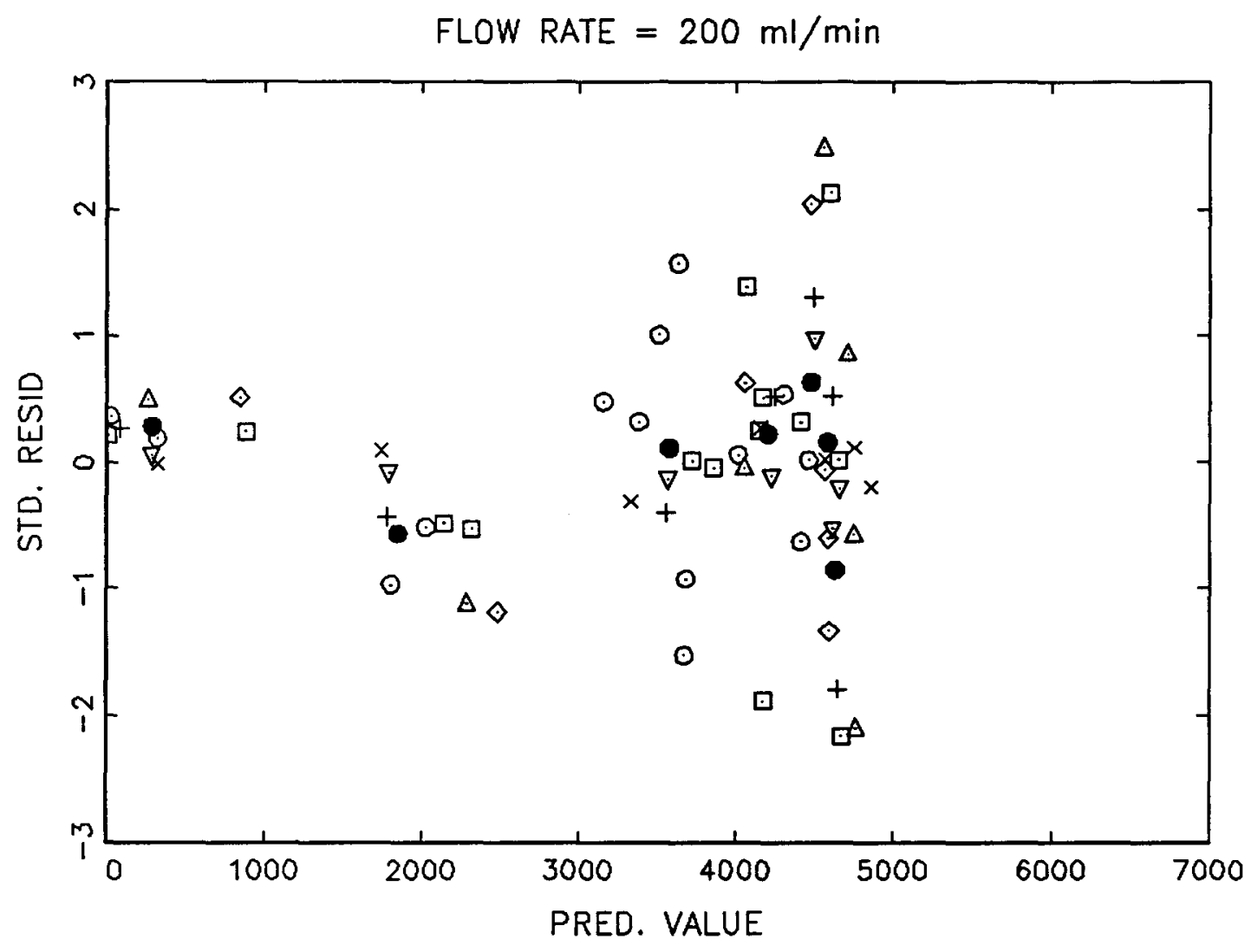

Fig. 3. 


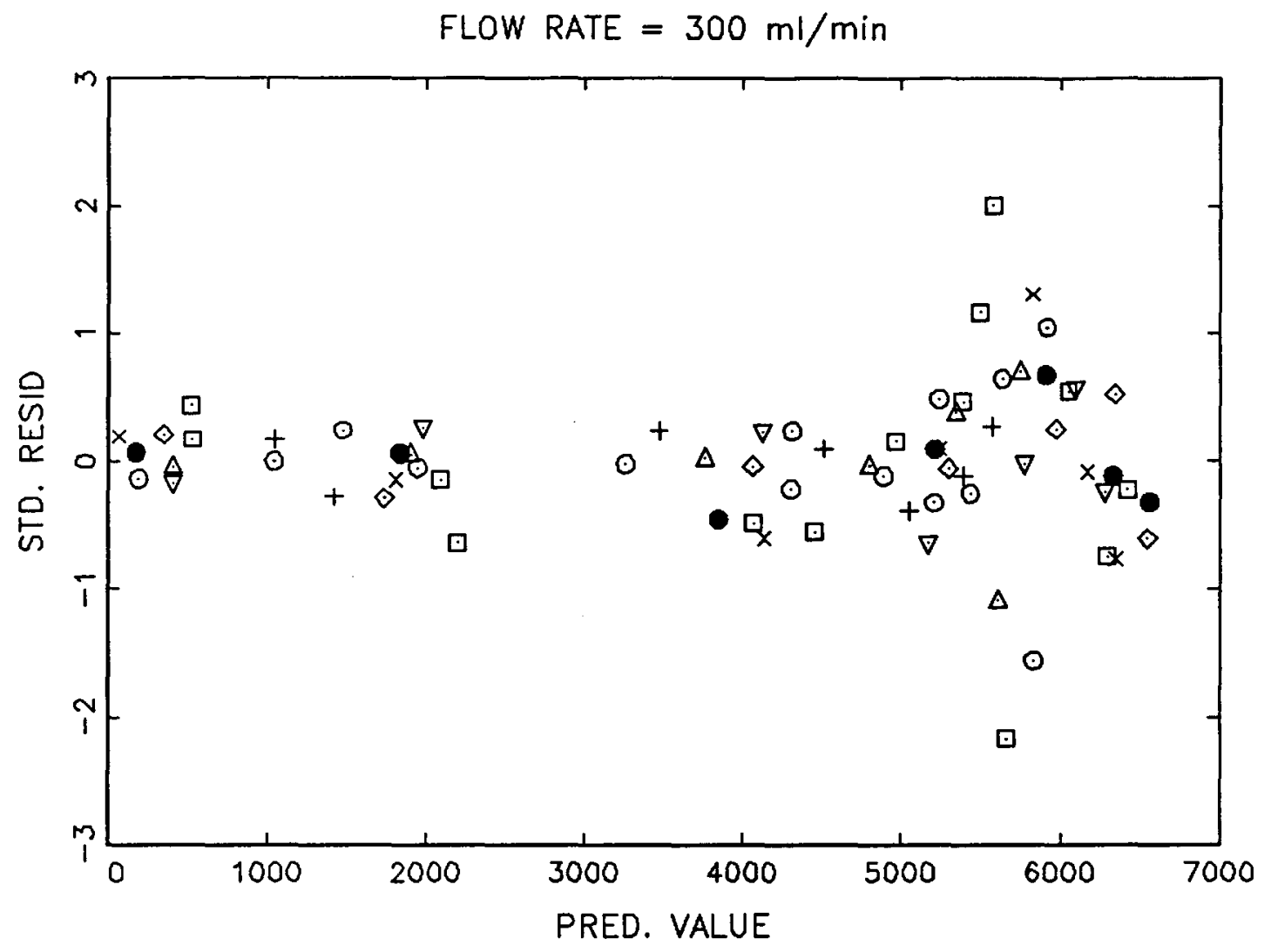




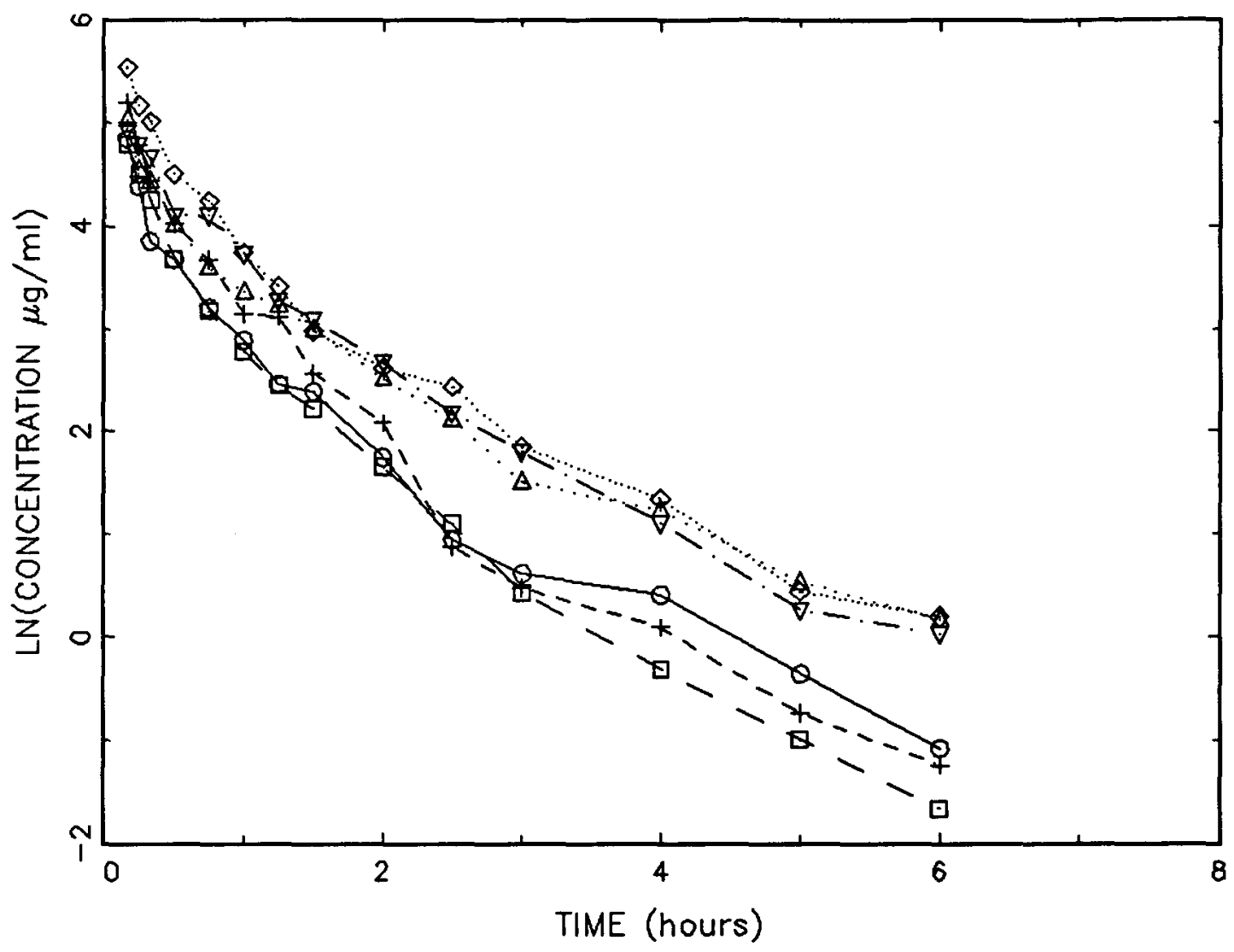

Fig. 4. 


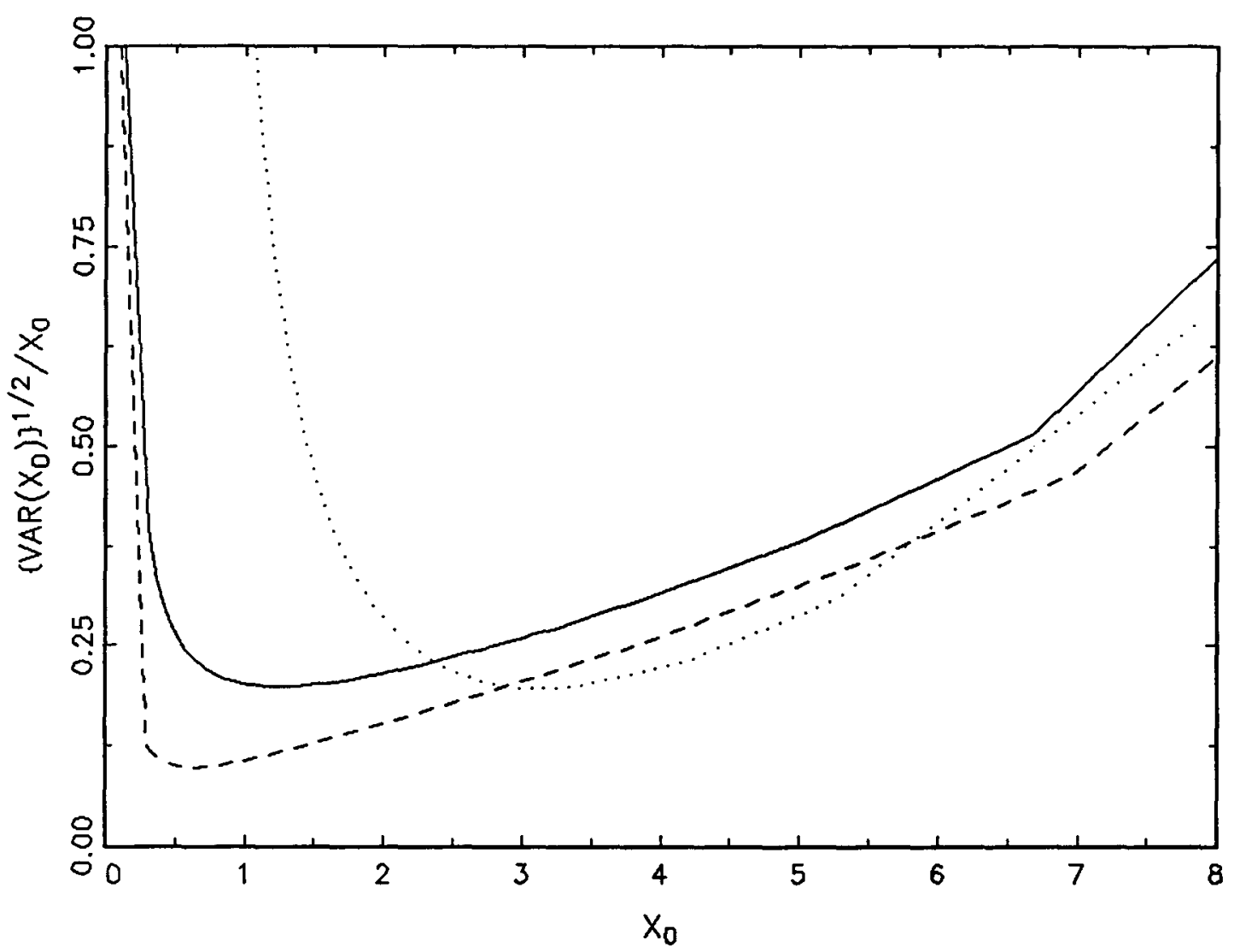

Fig. 5 . 


\section{TABLE 1}

Relaxin bioassay responses (pmoles/ml) for nine experiments; $x=$ standard concentration $(\mathrm{ng} / \mathrm{ml}$ ), triplicate responses at each $x$ except 0.0

\begin{tabular}{|c|c|c|c|}
\hline \multicolumn{4}{|c|}{ Experiment } \\
\hline $\mathbf{x}$ & 1 & 2 & 3 \\
\hline 0.00 & 1.77 & 1.80 & 1.87 \\
\hline 0.34 & $3.35,4.00,6.10$ & $4.20,5.85,4.70$ & $3.60,4.70,4.20$ \\
\hline 0.69 & $8.40,12.00,8.15$ & $7.10,9.20,9.00$ & $8.65,8.60,4.85$ \\
\hline 1.38 & $13.25,24.25,17.85$ & $15.00,23.45,25.40$ & $13.00,16.95,13.85$ \\
\hline 2.75 & $40.15,49.35,40.05$ & $30.15,46.90,74.45$ & $24.60,34.60,31.70$ \\
\hline 5.50 & $61.85,85.15,58.80$ & $51.85,72.95,83.70$ & $57.45,58.25,49.05$ \\
\hline 11.00 & $95.05,118.50,76.25$ & $66.30,101.25,92.80$ & $60.55,77.80,78.95$ \\
\hline 22.00 & $116.70,140.45,90.40$ & $87.50,115.30,109.40$ & $74.15,103.90,89.85$ \\
\hline \multicolumn{4}{|c|}{ Experiment } \\
\hline $\mathbf{x}$ & 4 & 5 & 6 \\
\hline 0.00 & 1.62 & 1.03 & 1.37 \\
\hline 0.34 & $3.65,3.60,4.95$ & $1.60,2.30,2.95$ & $3.95,4.00,3.75$ \\
\hline 0.69 & $5.35,7.35,9.00$ & $3.15,5.05,6.70$ & $6.00,10.60,10.00$ \\
\hline 1.38 & $10.80,18.25,19.60$ & $7.60,11.55,12.80$ & $16.20,23.95,22.30$ \\
\hline 2.75 & $21.75,26.05,32.60$ & $14.90,23.75,27.55$ & $34.80,61.75,54.05$ \\
\hline 5.50 & $57.05,92.05,99.90$ & $34.85,61.50,50.00$ & $62.25,112.90,105.60$ \\
\hline 11.00 & $85.10,92.30,104.90$ & $47.40,57.20,61.25$ & $99.30,137.15,155.10$ \\
\hline 22.00 & $87.55,97.35,101.60$ & $38.70,55.30,63.20$ & $101.95,137.15,155.10$ \\
\hline \multicolumn{4}{|c|}{ Experiment } \\
\hline $\mathbf{x}$ & 7 & 8 & 9 \\
\hline 0.00 & 1.17 & 1.87 & 2.32 \\
\hline 0.34 & $3.15,3.70,3.70$ & $5.40,4.55,3.05$ & $4.85,4.50,4.20$ \\
\hline 0.69 & $2.45,6.45,6.25$ & $7.35,7.10,7.30$ & $8.40,7.90,7.05$ \\
\hline 1.38 & $11.85,16.75,23.55$ & $11.10,16.35,14.15$ & $12.90,18.20,16.10$ \\
\hline 2.75 & $28.45,42.45,49.40$ & $25.70,27.85,35.90$ & $31.25,37.55,35.75$ \\
\hline 5.50 & $51.75,91.25,77.10$ & $49.30,63.60,72.95$ & $65.95,94.60,71.65$ \\
\hline 11.00 & $81.10,122.90,125.90$ & $57.50,71.45,69.75$ & $63.75,68.85,82.60$ \\
\hline 22.00 & $72.90,118.20,117.95$ & $69.65,92.05,88.55$ & $83.70,103.85,93.00$ \\
\hline
\end{tabular}


TABLE 2

Ultrafiltration rate responses $(\mathrm{ml} / \mathrm{hr})$ y for 20 high flux hemodialyzers operated at two flow rates; $\mathrm{x}=$ transmembrane pressure $(\mathrm{mmHg})$

\begin{tabular}{|c|c|c|c|c|c|c|c|c|}
\hline \multirow{2}{*}{$\frac{\text { Dialyzer }}{1}$} & \multicolumn{8}{|c|}{ Flow rate $=200 \mathrm{ml} / \mathrm{min}$} \\
\hline & y & 123.0 & 1537.5 & 3283.5 & 3783.0 & 4059.0 & 3255.0 & 3430.5 \\
\hline & $\mathbf{x}$ & 23.5 & 50.5 & 102.0 & 147.5 & 197.0 & 248.0 & 300.0 \\
\hline \multirow[t]{2}{*}{2} & $\mathbf{y}$ & 948.0 & 2175.0 & 3723.0 & 4443.0 & 4216.5 & 4306.5 & 3661.5 \\
\hline & $\mathbf{x}$ & 30.5 & 50.5 & 99.5 & 150.0 & 199.0 & 248.0 & 300.0 \\
\hline \multirow[t]{2}{*}{3} & $\mathbf{y}$ & 393.0 & 1983.0 & 4042.5 & 5225.0 & 4939.5 & 4597.5 & 4191.0 \\
\hline & $\mathbf{x}$ & 25.5 & 49.5 & 99.5 & 148.0 & 199.5 & 249.0 & 303.0 \\
\hline \multirow[t]{2}{*}{4} & $\mathbf{y}$ & 156.0 & 1665.0 & 3453.0 & 4381.5 & 4849.5 & 4752.0 & 4164.0 \\
\hline & $x$ & 25.0 & 49.5 & 100.0 & 150.0 & 196.5 & 248.5 & 298.0 \\
\hline \multirow[t]{2}{*}{5} & y & 982.5 & 2163.0 & 4227.0 & 5028.0 & 4551.0 & 4425.0 & 4230.0 \\
\hline & $\mathbf{x}$ & 30.5 & 50.5 & 98.0 & 150.5 & 200.5 & 250.5 & 299.0 \\
\hline \multirow[t]{2}{*}{6} & $\mathbf{y}$ & 298.5 & 1770.0 & 3529.5 & 4195.5 & 4761.0 & 4473.0 & 4603.5 \\
\hline & $\mathbf{x}$ & 24.5 & 48.0 & 101.0 & 150.5 & 200.0 & 251.5 & 297.0 \\
\hline \multirow[t]{2}{*}{7} & $\mathbf{y}$ & 321.0 & 1770.5 & 3249.0 & 4233.0 & 4573.5 & 4785.0 & 4804.5 \\
\hline & $\mathbf{x}$ & 25.5 & 51.5 & 100.0 & 150.5 & 202.0 & 249.0 & 301.0 \\
\hline \multirow{2}{*}{8} & $\mathbf{y}$ & 366.0 & 1695.0 & 3609.0 & 4263.0 & 4647.0 & 4627.5 & 4398.0 \\
\hline & $\mathbf{x}$ & 26.0 & 50.0 & 102.0 & 149.0 & 199.0 & 248.0 & 299.5 \\
\hline \multirow[t]{2}{*}{9} & $\mathbf{y}$ & 372.0 & 1888.5 & 3469.5 & 4030.5 & 4447.5 & 4243.5 & 4465.5 \\
\hline & $\mathbf{x}$ & 24.0 & 54.0 & 99.5 & 147.5 & 200.0 & 250.0 & 301.0 \\
\hline \multirow[t]{2}{*}{10} & $\mathbf{y}$ & 64.5 & 2011.5 & 3846.0 & 4498.5 & 5176.5 & 4657.5 & 4081.5 \\
\hline & $\mathbf{x}$ & 24.0 & 50.5 & 99.5 & 148.5 & 202.0 & 249.5 & 297.0 \\
\hline \multicolumn{4}{|l|}{ Dialyzer } & \multicolumn{5}{|c|}{ Flow rate $=300 \mathrm{ml} / \mathrm{min}$} \\
\hline \multirow[t]{2}{*}{1} & $y$ & 150.0 & 1540.5 & 3252.0 & 4243.5 & 4857.0 & 5368.5 & 5365.5 \\
\hline & $\mathbf{x}$ & 28.5 & 52.0 & 100.5 & 150.0 & 198.5 & 249.0 & 299.5 \\
\hline \multirow[t]{2}{*}{2} & $y$ & 642.0 & 2025.0 & 4305.0 & 5811.0 & 6199.5 & 6091.5 & 6360.0 \\
\hline & $\mathbf{x}$ & 29.5 & 51.5 & 101.0 & 148.0 & 200.0 & 248.0 & 300.5 \\
\hline \multirow[t]{2}{*}{3} & $y$ & 388.5 & 1915.5 & 3765.0 & 4789.5 & 5449.5 & 5317.5 & 5935.5 \\
\hline & $\mathbf{x}$ & 25.5 & 50.0 & 98.0 & 149.0 & 201.5 & 251.0 & 298.0 \\
\hline \multirow[t]{2}{*}{4} & $y$ & 1093.5 & 1347.0 & 3535.5 & 4534.5 & 4944.0 & 5362.5 & 5643.0 \\
\hline & $\mathbf{x}$ & 40.0 & 47.0 & 101.0 & 151.5 & 198.0 & 251.0 & 300.0 \\
\hline \multirow[t]{2}{*}{5} & $y$ & 405.0 & 1659.0 & 4051.5 & 5284.5 & 6043.5 & 6483.0 & 6382.5 \\
\hline & $\mathbf{x}$ & 29.0 & 49.5 & 101.5 & 152.0 & 202.0 & 250.0 & 297.5 \\
\hline \multirow[t]{2}{*}{6} & $y$ & 360.0 & 2049.0 & 4188.0 & 4999.5 & 5767.5 & 6247.5 & 6214.5 \\
\hline & $\mathbf{x}$ & 23.5 & 48.0 & 101.0 & 149.0 & 199.0 & 248.0 & 300.5 \\
\hline \multirow[t]{2}{*}{7} & $y$ & 117.0 & 1768.5 & 3970.5 & 5268.0 & 6180.0 & 6148.5 & 6142.5 \\
\hline & $\mathbf{x}$ & 23.5 & 48.5 & 102.5 & 151.5 & 199.0 & 251.0 & 302.0 \\
\hline \multirow[t]{2}{*}{8} & $\mathbf{y}$ & 189.0 & 1851.0 & 3721.5 & 5235.0 & 6091.5 & 6298.5 & 6477.0 \\
\hline & $\mathrm{x}$ & 26.0 & 51.5 & 97.0 & 150.5 & 199.0 & 250.0 & 299.5 \\
\hline \multirow[t]{2}{*}{9} & $y$ & 1041.0 & 1932.0 & 4377.0 & 5122.5 & 5809.5 & 5409.0 & 6201.0 \\
\hline & $\mathrm{x}$ & 35.5 & 48.0 & 102.5 & 150.0 & 199.0 & 250.0 & 300.5 \\
\hline \multirow[t]{2}{*}{10} & $\mathbf{y}$ & 571.5 & 2050.5 & 3940.5 & 5010.0 & 5515.5 & 6118.5 & 5071.5 \\
\hline & $x$ & 28.0 & 50.5 & 100.0 & 149.0 & 200.0 & 250.5 & 302.0 \\
\hline
\end{tabular}


TABLE 3

Plasma concentrations $(\mu \mathrm{g} / \mathrm{ml})$ of cefamandole following 10 minute infusion in 6 subjects

\begin{tabular}{|c|c|c|c|c|c|c|}
\hline \multirow{2}{*}{$\begin{array}{l}\text { Time } \\
(\min )\end{array}$} & \multicolumn{6}{|c|}{ Subject } \\
\hline & 1 & 2 & 3 & 4 & 5 & 6 \\
\hline 10 & 127.00 & 120.00 & 154.00 & 181.00 & 253.00 & 140.00 \\
\hline 15 & 807.00 & 90.10 & 94.00 & 119.00 & 176.00 & 120.00 \\
\hline 20 & 47.40 & 70.00 & 84.00 & 84.30 & 150.00 & 106.00 \\
\hline 30 & 39.90 & 40.10 & 56.00 & 56.10 & 90.30 & 60.40 \\
\hline 45 & 24.80 & 24.00 & 37.10 & 39.80 & 69.60 & 60.90 \\
\hline 60 & 17.90 & 16.10 & 28.90 & 23.30 & 42.50 & 42.20 \\
\hline 75 & 11.70 & 11.60 & 25.20 & 22.70 & 30.60 & 26.80 \\
\hline 90 & 10.90 & 9.20 & 20.00 & 13.00 & 19.60 & 22.00 \\
\hline 120 & 5.70 & 5.20 & 12.40 & 8.00 & 13.80 & 14.50 \\
\hline 150 & 2.55 & 3.00 & 8.30 & 2.40 & 11.40 & 8.80 \\
\hline 180 & 1.84 & 1.54 & 4.50 & 1.60 & 6.30 & 6.00 \\
\hline 240 & 1.50 & 0.73 & 3.40 & 1.10 & 3.80 & 3.00 \\
\hline 300 & 0.70 & 0.37 & 1.70 & 0.48 & 1.55 & 1.30 \\
\hline 360 & 0.34 & 0.19 & 1.19 & 0.29 & 1.22 & 1.03 \\
\hline
\end{tabular}


TABLE 4

Estimation based on individual and pooled data, relaxin bioassay data (standard errors for individual GLS regression parameter estimates based on standard theory are given in parentheses)

$$
\begin{array}{cccccccccccc}
\multicolumn{3}{l}{\text { LS, Individual Data }} & \multicolumn{4}{c}{\text { GLS-PL, Individual Data }} & \multicolumn{3}{c}{\text { GLS-PL, Pooled Data }} \\
\beta_{1} & \beta_{2} & \beta_{3} & \beta_{4} & \beta_{1} & \beta_{2} & \beta_{3} & \beta_{4} & \beta_{1} & \beta_{2} & \beta_{3} & \beta_{4}
\end{array}
$$

\begin{tabular}{|c|c|c|c|c|c|c|c|c|c|c|c|c|}
\hline 1 & 132.58 & 0.71 & 1.64 & 1.31 & $\begin{array}{c}127.37 \\
(16.59)\end{array}$ & $\begin{array}{r}1.76 \\
(0.44) \\
0.27\end{array}$ & $\begin{array}{l}1.57 \\
(0.23) \\
0.93\end{array}$ & $\begin{array}{c}1.43 \\
(0.14)\end{array}$ & $\begin{array}{c}127.32 \\
(19.33)\end{array}$ & $\begin{array}{l}1.76 \\
(0.36)\end{array}$ & $\begin{array}{l}1.57 \\
(0.26)\end{array}$ & $\begin{array}{l}1.43 \\
(0.14)\end{array}$ \\
\hline 2 & 111.05 & -1.03 & 1.27 & 1.31 & $\begin{array}{c}107.79 \\
(16.38)\end{array}$ & $\begin{array}{c}1.86 \\
(0.29) \\
0.14\end{array}$ & $\begin{array}{l}1.27 \\
(0.24) \\
1.16\end{array}$ & $\begin{array}{l}1.55 \\
(0.16)\end{array}$ & $\begin{array}{c}105.56 \\
(12.80)\end{array}$ & $\begin{array}{l}1.90 \\
(0.38)\end{array}$ & $\begin{array}{l}1.23 \\
(0.21)\end{array}$ & $\begin{array}{l}1.59 \\
(0.16)\end{array}$ \\
\hline 3 & 100.12 & 1.60 & 1.64 & 1.38 & $\begin{array}{c}100.76 \\
(10.37)\end{array}$ & $\begin{array}{c}1.93 \\
(0.35) \\
0.21\end{array}$ & $\begin{array}{l}1.66 \\
(0.19) \\
0.90\end{array}$ & $\begin{array}{c}1.39 \\
(0.11)\end{array}$ & $\begin{array}{c}101.83 \\
(17.11)\end{array}$ & $\begin{array}{l}1.91 \\
(0.38)\end{array}$ & $\begin{array}{l}1.67 \\
(0.29)\end{array}$ & $\begin{array}{l}1.38 \\
(0.15)\end{array}$ \\
\hline 4 & 95.96 & 7.65 & 1.30 & 4.18 & $\begin{array}{c}127.86 \\
(27.77)\end{array}$ & $\begin{array}{c}1.68 \\
(0.28) \\
0.16\end{array}$ & $\begin{array}{l}1.75 \\
(0.33) \\
1.16\end{array}$ & $\begin{array}{l}1.43 \\
(0.15)\end{array}$ & $\begin{array}{l}121.58 \\
(18.74)\end{array}$ & $\begin{array}{l}1.76 \\
(0.38)\end{array}$ & $\begin{array}{l}1.66 \\
(0.25)\end{array}$ & $\begin{array}{l}1.49 \\
(0.15)\end{array}$ \\
\hline 5 & 54.67 & 4.11 & 1.15 & 3.25 & $\begin{array}{c}61.79 \\
(8.37)\end{array}$ & $\begin{array}{r}1.07 \\
(0.27) \\
0.27\end{array}$ & $\begin{array}{l}1.29 \\
(0.22) \\
0.99\end{array}$ & $\begin{array}{l}1.65 \\
(0.20)\end{array}$ & $\begin{array}{c}62.13 \\
(7.51)\end{array}$ & $\begin{array}{l}1.06 \\
(0.21)\end{array}$ & $\begin{array}{c}1.30 \\
(0.20)\end{array}$ & $\begin{array}{c}1.64 \\
(0.16)\end{array}$ \\
\hline 6 & 158.99 & 1.85 & 1.50 & 1.67 & $\begin{array}{c}165.58 \\
(25.50)\end{array}$ & $\begin{array}{c}1.37 \\
(0.14) \\
0.10\end{array}$ & $\begin{array}{l}1.58 \\
(0.21) \\
1.21\end{array}$ & $\begin{array}{l}1.57 \\
(0.10)\end{array}$ & $\begin{array}{c}164.12 \\
(22.62)\end{array}$ & $\begin{array}{l}1.38 \\
(0.28)\end{array}$ & $\begin{array}{l}1.56 \\
(0.21)\end{array}$ & $\begin{array}{l}1.58 \\
(0.13)\end{array}$ \\
\hline 7 & 111.08 & 3.21 & 1.33 & 2.12 & $\begin{array}{c}118.09 \\
(16.07)\end{array}$ & $\begin{array}{c}1.43 \\
(0.44) \\
0.35\end{array}$ & $\begin{array}{l}1.42 \\
(0.21) \\
0.93\end{array}$ & $\begin{array}{l}1.74 \\
(0.21)\end{array}$ & $\begin{array}{c}121.39 \\
(15.53)\end{array}$ & $\begin{array}{l}1.34 \\
(0.49)\end{array}$ & $\begin{array}{l}1.47 \\
(0.21)\end{array}$ & $\begin{array}{l}1.68 \\
(0.15)\end{array}$ \\
\hline 8 & 82.64 & 3.07 & 1.31 & 1.86 & $\begin{array}{l}91.93 \\
(10.71)\end{array}$ & $\begin{array}{c}2.08 \\
(0.41) \\
0.22\end{array}$ & $\begin{array}{l}1.52 \\
(0.21) \\
0.95\end{array}$ & $\begin{array}{l}1.47 \\
(0.15)\end{array}$ & $\begin{array}{l}93.83 \\
(14.44)\end{array}$ & $\begin{array}{l}2.02 \\
(0.40)\end{array}$ & $\begin{array}{l}1.56 \\
(0.27)\end{array}$ & $\begin{array}{l}1.44 \\
(0.16)\end{array}$ \\
\hline 9 & 86.19 & 5.51 & 1.15 & 2.73 & $\begin{array}{c}111.69 \\
(17.42)\end{array}$ & $\begin{array}{r}2.35 \\
(0.16) \\
0.03\end{array}$ & $\begin{array}{l}1.61 \\
(0.21) \\
1.47\end{array}$ & $\begin{array}{l}1.47 \\
(0.08)\end{array}$ & $\begin{array}{l}98.44 \\
(12.79)\end{array}$ & $\begin{array}{l}2.56 \\
(0.49)\end{array}$ & $\begin{array}{l}1.37 \\
(0.21)\end{array}$ & $\begin{array}{l}1.64 \\
(0.19)\end{array}$ \\
\hline & & & & & & & & & \multicolumn{4}{|c|}{$\begin{array}{l}\text { Pooled estimate of } \sigma=0.2 \\
\text { Pooled estimate of } \theta=1.03\end{array}$} \\
\hline
\end{tabular}

Experiment 
TABLE 5

Analysis of the cefamandole data: (a) Results using the GLS and GTS algorithms of Section 4.2 (b) Results using the GLS algorithm of Section 4.3 (after 3 iterations)

Elements of $\beta_{i}^{*}$ : GLS-PL, pooled data Subject (a)

Elements of $\hat{\beta}_{i}$ : "Refined" GTS estimates

$$
\begin{array}{llll}
\beta_{1} & \beta_{2} & \beta_{3} & \beta_{4}
\end{array}
$$

Implied CVs for kinetic parameters (\%):

$$
[6.9,26.6,6.0,186.8]^{\mathrm{T}}
$$

Elements of $\hat{\hat{\beta}}_{i}:$ GLS algorithm with PL

\begin{tabular}{|c|c|c|c|c|}
\hline & $\beta_{1}$ & $\beta_{2}$ & $\beta_{3}$ & $\beta_{4}$ \\
\hline 1 & 5.500 & 1.836 & 3.778 & -0 . \\
\hline & 5.256 & 1.482 & 3.665 & -0 \\
\hline & 5.489 & 1.685 & 3.961 & -0.3 \\
\hline & 5.819 & 1.876 & 4.231 & 0.0 \\
\hline & 5.8 & 1.207 & 4.280 & 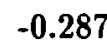 \\
\hline & 4.947 & 1.350 & 4.356 & -0.1 \\
\hline
\end{tabular}

(b)

$$
\begin{gathered}
\hat{\gamma}=[5.484,1.584,4.130,-0.169]^{\mathrm{T}} \\
\hat{\Sigma}=\left[\begin{array}{cccc}
0.077 & -0.010 & 0.001 & 0.004 \\
\cdot & 0.030 & -0.054 & 0.011 \\
\cdot & \cdot & 0.054 & -0.017 \\
\cdot & \cdot & \cdot & 0.012
\end{array}\right]
\end{gathered}
$$

Estimate of $\sigma=0.54$

Estimate of $\theta=0.58$

Implied CVs for kinetic parameters (\%):

$$
[5.1,10.9,5.6,64.8]^{\mathrm{T}}
$$

